\title{
DISCRETE AND CONTINUUM RELAXATION DYNAMICS OF FACETED CRYSTAL SURFACE IN EVAPORATION MODELS*
}

\author{
KANNA NAKAMURA ${ }^{\dagger}$ AND DIONISIOS MARGETIS $\ddagger$
}

\begin{abstract}
We study linkages of two scales in the relaxation of an axisymmetric crystal with a facet in evaporation-condensation kinetics. The macroscale evolution is driven by the motion of concentric circular, repulsively interacting line defects (steps) which exchange atoms with the vapor. At the microscale, the step velocity is proportional to the variation of the total step free energy, leading to large systems of differential equations for the step radii. We focus on two step flow models. In one model (called M1) the discrete mobility is simply proportional to the upper-terrace width; in another model (M2) the mobility is altered by an extra geometric factor. By invoking self-similarity at long time, we numerically demonstrate that (i) in M1, discrete slopes follow closely a continuum thermodynamics approach with "natural boundary conditions" at the facet edge; (ii) in contrast, predictions of M2 deviate from results of the above continuum approach; and (iii) this discrepancy can be eliminated via a continuum boundary condition with a geometry-induced jump for top-step collapses. At the macroscale, both step models give rise to free-boundary problems for a second-order, parabolic partial differential equation, which we study via the subgradient formalism. We discuss the interpretation of the facet height as shock and prove convergence of the solution of each discrete scheme to the (weak) entropy solution of a conservation law if steps do not interact.
\end{abstract}

Key words. crystal surface, evaporation, facet, Burton-Cabrera-Frank model, subgradient formalism, free-boundary problem, shock

AMS subject classifications. 74A50, 35Q99, 35R35

DOI. $10.1137 / 110849687$

1. Introduction. Crystal surface structures may relax to become flat in the absence of external material deposition, so that the large-scale height profile evolves by the variation of thermodynamics-based free energies [4, 21, 27, 39, 51]. Below the roughening transition, this evolution must be driven by the motion of atomic line defects (steps) separated by nanoscale terraces [5, 26]. Presently, the relation of step motion to surface morphological relaxation is inadequately understood.

In particular, crystals develop macroscopic plateaus - facets. Away from facets, surface relaxation can be described by partial differential equations (PDEs) for the height or slope profiles consistent with steps and subject to variational and conservation principles; for derivations of PDEs from step flow, see, e.g., [9, 35, 41, 43, 46, 51]. This description in principle breaks down near facets; extremal-step collapses can affect the surface profile macroscopically, as pointed out in $[6,24,34,50]$. The incorporation of facets into computationally appealing continuum theories in realistic geometries is a challenging and rich problem.

In this article, we study via numerics the relation of step flow to its continuum limit in a radial geometry with a facet under evaporation-condensation kinetics, where circular, descending steps move by exchange of atoms with the vapor. At the

*Received by the editors September 29, 2011; accepted for publication (in revised form) November 20, 2012; published electronically January 29, 2013.

http://www.siam.org/journals/mms/11-1/84968.html

${ }^{\dagger}$ Department of Mathematics, University of Maryland, College Park, MD 20742-4015 (nakamura@ math.umd.edu). This author's research was supported by a Graduate Student Summer Research Fellowship during the summer of 2011 and by NSF DMS-0847587 at the University of Maryland.

${ }^{\ddagger}$ Department of Mathematics, and Institute for Physical Science and Technology, and Center for Scientific Computation and Mathematical Modeling, University of Maryland, College Park, MD 20742-4015 (dio@math.umd.edu). This author's research was supported by NSF DMS-0847587 at the University of Maryland. 
macroscale outside the facet, we invoke a second-order parabolic PDE proposed by Spohn [51]. This PDE has a conservation form and a simple variational characterization: the height flow equals the first variation of a convex, singular free energy. We treat the facet as a free boundary. One choice is to apply "natural boundary conditions" from the subgradient formalism which is intimately connected to continuum thermodynamics [27]. ${ }^{1}$ At the microscale, we propose two step flow models, called M1 and M2, each of which formally reduces to Spohn's PDE away from the facet. In both models, the step velocity is driven by the step chemical potential, the variation of the total step free energy. In M1 the step mobility is simply proportional to the upper-terrace width, whereas in M2 the mobility is modified by an extra discrete geometry-induced factor. Our numerics under conical initial data indicate that the discrete slopes become self-similar at long time. In this regime, we mainly find that

(i) discrete slopes by M1 are in agreement with predictions of the subgradient theory;

(ii) discrete slopes by M2 deviate from results of the subgradient formalism; and

(iii) the above discrepancy is eliminated by introduction of a jump at the facet edge of a (properly defined) continuum flux function, which replaces the natural boundary condition that this flux be continuous.

The modification of the natural boundary condition in (iii) aims to account for the facet microstructure. The discontinuity is expressed in terms of the extra, discrete geometric factor of step mobility evaluated for a pair of extremal steps at times of top-step collapses; data for this factor is extracted from discrete simulations. In the special case with noninteracting steps in M2, we verify that this factor has a value close to an exact result from the convergence of the discrete scheme.

These results indicate that, depending on step kinetics, facets may be incorporated into a continuum theory by adjusting a coefficient in a known (natural) boundary condition of the subgradient formalism. This finding essentially justifies the use of this formalism as our starting point. For nonzero step-step interactions, the natural boundary conditions include continuity of height, slope, and the flux entering the conservation form of the PDE outside the facet. Retaining these variables, we point out that adjusting the flux at the facet edge in correspondence to collapses of atomic layers suffices to yield continuum predictions in agreement with discrete simulations.

This viewpoint of ours is different from previous studies [24, 34], which focus on facet evolution under (the more complicated) surface diffusion in radial geometries, where the step velocity is determined by mass fluxes of adsorbed atoms (adatoms) impinging on each step. In these studies [24, 34], one of the natural boundary conditions, the continuity of the large-scale step chemical potential, is replaced by a statement for the facet speed accounting for individual step collapses on the facet. This modification is puzzling: it seems unrelated to continuum thermodynamics, cannot be clearly transferred to non-self-similar settings, and is expressed by high derivatives of the height which render surface profiles sensitive to any error in the collapse times.

Seeking an alternate approach, we focus on evaporation kinetics under selfsimilarity of slopes. This restriction enables us to single out the effect of key parameters with relative computational ease. We deem that our proposal for a discontinuity in a natural boundary condition has advantages over $[24,34]$ such as (a) the condition can plausibly be extended to all times (when self-similarity may not hold) and (b) the jump depends only on the first derivative of height (slope) and a suitable factor.

\footnotetext{
${ }^{1}$ The subgradient theory will also be called the "continuum thermodynamics approach" here.
} 
However, our modification of the natural boundary condition is phenomenological. The derivation of the jump directly from step flow is largely unexplored.

Our broader goal is to shed light on the linkage of continuum thermodynamics to underlying discrete schemes when the number of steps is not preserved, e.g., if step collapses occur on top of facets. It is tempting to expect that this feature should always imply lack of consistency of the discrete system with the continuum thermodynamics approach $[24,25]$. We numerically show that this connection depends critically on the step kinetics (particularly the step velocity law); for example, the subgradient theory is in agreement with step flow in the context of M1.

Notably, most but by no means all $[28,51]$ previous studies of facet evolution in one-dimensional (1D) or radial geometries focus on surface diffusion in the absence of evaporation; see, e.g., [1, 2, 24, 25, 34]. In [28], the authors point out the significant influence of kinetics, especially nucleation, on the connection of continuum laws (i.e., the Hamilton-Jacobi equation for the height) to step motion near facets.

Physically, evaporation coexists with surface diffusion; the latter process, in which adatoms diffuse on terraces and attach or detach at steps from or to terraces [5, 26], becomes dominant for very small structures $[20,45]$. To capture key elements in the relation of discrete step flow and continuum dynamics, we consider evaporation models (M1 and M2) that are rich enough to include step curvature, elastic-dipole step-step repulsions [32, 40], and a terrace-width-dependent step mobility. For M2, we construct the geometry-induced step mobility with recourse to a special limit of a BurtonCabrera-Frank-(BCF-) type model for circular steps under the combined effects of desorption, diffusion of adatoms on terraces, and attachment/detachment of atoms at steps with negative ("inverse") Ehrlich-Schwoebel (ES) barrier [10, 44, 47, 49] by which the sticking kinetic rate at down-steps is larger than that for up-steps.

Our work has limitations. We assume that the continuum limit exists for interacting steps and submit a proof for such a limit only for noninteracting steps (see section 7). We only provide numerical evidence that solutions of the discrete schemes for M1 and M2 under self-similarity may converge to certain continuum-scale solutions, with the exception of the special case with zero step interactions, which we discuss analytically. The numerically observed self-similar behavior of discrete and continuum slopes is not explored rigorously. Our jump condition for interacting steps requires input from discrete simulations; it is an open problem to formulate alternate conditions without use of step collapse times. The full time-dependent problem (in absence of self similarity) is not studied. Our step models should be enriched to include more realistic effects such as terrace diffusion and nonradial geometries; the effect of terrace diffusion is outlined in Appendix A.

We assume that the reader is familiar with the fundamentals of epitaxial growth. For broad reviews on the subject, see, e.g., [26, 37, 38].

1.1. Microscopic models. Three features of our evaporation models, which partly motivate their use here, are (i) compatibility with Spohn's PDE outside facets [27, 51]; (ii) well-posedness of the ensuing discrete dynamics even for zero step-step interaction where M1 and M2 become simple backward difference schemes; and (iii) numerically observed self-similar behavior of the discrete slopes at long time.

We start with a step velocity law of the form

$$
\frac{\mathrm{d} r_{i}}{\mathrm{~d} t}=\dot{r}_{i}=-\nu_{i}\left(\mu_{i}-\mu^{0}\right), \quad i=0,1 \ldots, N,
$$

which captures the effect of adatoms being exchanged between concentric circular step edges and the surrounding vapor; $N \gg 1$. In (1.1), $r_{i}(t)$ is the $i$ th-step radius, $\nu_{i}$ is 
a (positive) discrete mobility (specified below), $\mu^{0}$ is the constant chemical potential of the surrounding vapor, and $\mu_{i}$ is the step chemical potential; this $\mu_{i}$ incorporates step curvature (stiffness) and elastic-dipole step-step interactions [26] and depends on $\left(r_{i-1}, r_{i}, r_{i+1}\right)$ (see section 2$)$. We then obtain a large system of ordinary differential equations (ODEs) for the step radii. This system describes successive annihilations of the top (extremal) layers of an axisymmetric structure; each collapse time $t_{n}$ is defined as the earliest time at which $r_{n}(t)=0(n \geq 0)$. We take $r_{i}(t) \equiv 0$ if $i \leq n$ and $t \geq t_{n}$. Because of these annihilations, the surface height is expected to decrease with time. For definiteness, set $\mu^{0}=0$ in (1.1).

A few comments on the physical origin of (1.1) are in order. The guiding principle is that atoms on step edges move from higher to lower chemical potential. For example, when the top circular step is small enough, its chemical potential $\left(\mu_{i}\right)$ is dominated by the step line tension and tends to become large; thus, atoms leave the step edge and this in turn retreats (and shrinks). This behavior is consistent with (1.1).

The associated step mobility reads

$$
\nu_{i}=\nu \mathcal{G}_{i} \frac{r_{i}-r_{i-1}}{a} ; \quad \mathcal{G}_{i}=\mathcal{G}_{i}(t)=\left\{\begin{array}{lr}
1 & \text { for M1 } \\
\frac{r_{i}+r_{i-1}}{2 r_{i}} & \text { for M2 },
\end{array}\right.
$$

where $a$ is the step height and $\nu$ is a positive constant $(\nu>0)$. In section 3 , we show that if steps are ordered with $r_{i}(0)>r_{i-1}(0)$ for all $i$, then the ordering is preserved by the flow; thus, $\nu_{i}>0$ for all times $t_{i}>t>0$. The position-dependent formula for $\mathcal{G}_{i}$ in $\mathrm{M} 2$ can be derived with recourse to a special limit of the BCF model with desorption [5, 45], as outlined in Appendix A.

The quantity $\mathcal{G}_{i}$ forms a crucial geometric factor in M2. Note that step velocity law (1.1) for M2 reduces to the one for M1 away from the facet where we expect that $r_{i+1}-r_{i} \ll r_{i}, r_{i+1}$, a necessary condition for the (formal) continuum limit to make sense. This condition should break down at extremal steps, near the facet.

1.2. Continuum limit. In our axisymmetric setting, the facet is a macroscopic surface region of spatially constant height (zero slope) and growing radius, $r_{\mathrm{f}}(t)$. In the limit of a large number of steps, ODE system (1.1) with (1.2) formally reduces to Spohn's evolution PDE [51] for the height profile away from the facet. This PDE has the conservation form

$$
\partial_{t} h+c \operatorname{div} \boldsymbol{\xi}=0, \quad r>r_{\mathrm{f}}(t),
$$

where $h(r, t)$ is the height profile, $\boldsymbol{\xi}(r, t)$ is a vector-valued flux (defined in section 4 ) in the radial direction, $r$ is the polar coordinate, and $c$ is a positive constant. In the case of evaporation-condensation, $\boldsymbol{\xi}$ expresses mass exchange between the surface and its surrounding vapor and $\operatorname{div} \boldsymbol{\xi}$ is the continuum-scale step chemical potential.

By (1.3), the height flow outside the facet reads $\partial_{t} h \propto-\delta E / \delta h$, the first variation (in the $L^{2}$-metric) of the convex, singular free energy $[18,51]$

$$
E(h)=\iint\left(g_{1}|\nabla h|+\frac{g_{3}}{3}|\nabla h|^{3}\right) \mathrm{d} A,
$$

where $g_{1}$ is the step line tension and $g_{3}$ is the step-step interaction strength $\left(g_{1}>0\right.$, $g_{3} \geq 0$ ), and integration is carried out on the crystal reference $(x y-)$ plane. Notably, $\delta E / \delta h$ is ill-defined (in the usual local sense) on the facet $(\nabla h=0)$.

Rigorous study of the continuum limit yielding (1.3) for arbitrary $g_{3} / g_{1}>0$ lies beyond our scope. This limit is brought about by taking $a \rightarrow 0$ with fixed slope. We 
assume that the width of each terrace is much smaller than the radii of its bounding steps and varies slowly with the polar coordinate [35].

1.2.1. Subgradient formalism and free-boundary conditions. Our choice to use the subgradient formalism as a starting point at the continuum level is almost compelling: this theory is well-studied and incorporates facets into the continuum by treating the height flow as a dynamical system, without the need of input from the microscale [27]. Remarkably, the subgradient theory includes the facet in evolution by dictating that everywhere $\partial_{t} h$ is an appropriate element of the $L^{2}$-subdifferential (a nonempty set) of $E(h)$ [27]. Accordingly, PDE (1.3) is replaced by a similar statement valid for all $r \geq 0$; see section 5.1. The associated flux $\boldsymbol{\xi}$ is thus continuously extended onto the facet. In addition, the height is continuous across the facet edge, and for nonzero step interactions $\left(g_{3}>0\right)$ the surface slope is continuous.

Alternatively, the facet can be treated as a free boundary by enforcement of Spohn's PDE away from the facet [51]. This view is equivalent with the subgradient theory if the above three continuity relations, natural boundary conditions, are applied. ${ }^{2}$ The emerging recipe is simple: apply (1.3) and impose the three continuity conditions at the facet edge. This view, which reconciles continuum thermodynamics with the free-boundary approach, lies at the heart of our study.

By invoking self-similarity of the slope (see below), we numerically show that the above familiar viewpoint is consistent with the dynamics of M1 but needs to be amended for M2. For consistency with M2, we impose the condition that the flux $\boldsymbol{\xi}$ be discontinuous across the facet boundary; a novelty is to introduce a jump in terms of values of $\mathcal{G}_{i}(t)$, given in (1.2), at times $t \simeq t_{n}$ of step collapses (when $r_{n}(t)=0$ ) for the first two (extremal) steps of radii $r_{n+1}$ and $r_{n+2}$ (for $i=n+2$ in $\mathcal{G}_{i}$ ); see section 5.2. The ensuing continuum slope is found in excellent agreement with step simulations.

1.2.2. Self-similarity and boundary layer theory. For noninteracting steps $\left(g_{3}=0\right)$ and an initial linear cone, the continuum-scale slope, $m(r, t)$, and facet radius are determined exactly in closed form and have a self-similar structure. In addition, our step simulations for $g_{3} \geq 0$ suggest that the discrete slopes become self-similar at long time. Motivated by these observations and the need to circumvent complications of solving the full PDE, we apply a self-similarity ansatz to $m(r, t)$ away from the facet. This hypothesis is compatible with the structure of the evolution PDE for $m$ and the free-boundary conditions. In particular, for large $t$ we assume that $m(r, t) \sim \mathfrak{m}(\eta)$, where $\eta=r / \sqrt{t}>\eta_{\mathrm{f}}=r_{\mathrm{f}} / \sqrt{t}$; formally reduce the PDE to an ODE for $\mathfrak{m}(\eta)$; and solve this ODE numerically (see section 6).

To gain some analytical insight into continuum solutions, we apply boundary layer theory to the PDE for $m$ if step interactions are weak, $0<g_{3} / g_{1} \ll 1$; see section 6.3. Our approximation for $m$ indicates how the zero slope of the facet can be connected smoothly to the constant slope (for conical initial data) far from the facet and serves as a basis for some of our numerical computations.

Our study of self-similar solutions has limitations. First, we have not shown rigorously that the discrete slopes become self-similar, and the stability is not investigated. Second, the reduction of the PDE to a similarity ODE is only postulated. Third, we have not studied initial data other than a linear cone.

1.2.3. Facet as shock and convergence of discrete schemes. In light of our findings, we discuss the connection of our discrete schemes for M1 and M2 to

\footnotetext{
${ }^{2}$ In [51], Spohn derives these boundary conditions via a nonlinear transformation, by analogy with PDE theory for porous media and nonlinear filtration, without direct use of the subgradient.
} 
continuum-scale shocks particularly for zero step interactions $\left(g_{3}=0\right)$. For this purpose, we write the evolution PDE outside the facet in Lagrangian variables; the step position, $R$, is viewed as a function of height, $h$, and time [12]. First, we posit that the facet is related to a shock wave in the context of distinct conservation laws for functions of $R$ if $g_{3}=0$ (see section 7.1); the shock speed and strength are directly related to the facet speed and radius, respectively.

We then consider M1 and M2 as discretizations of respective weak formulations for distinct conservation laws. In fact, we prove that the discrete solution of each model converges to the entropy solution of a conservation law (see section 7.2); this limit must be the above shock wave. In this context, the boundary condition for the flux $\boldsymbol{\xi}$ is viewed as a Rankine-Hugoniot-type condition from the corresponding weak formulation; $R$ is zero on the one side of the shock, as implied by the initial data for step radii. In this vein, we exactly evaluate the jump of $\boldsymbol{\xi}$ for M2.

This is not the first time facets are connected to shocks; see [12, 13, 14, 15, 16, 52] where PDE solutions are recognized as shocks. We adopt the shock notion with a different perspective: to link this picture to step schemes. We are able to establish indirectly a connection of the subgradient theory to schemes for noninteracting steps. However, our discussion (section 7) is incomplete at the moment since it does not rigorously address step interactions.

1.3. On past works. The connection of step flow to continuum theories has been studied analytically for semi-infinite 1D facets at fixed heights in surface diffusion [1, 2, 36]; however, only the attachment-detachment limited (ADL) regime has been addressed rigorously $[1,2]$. In this setting, the surface height is a convenient independent variable by which there is no need to use a free boundary for the facet; furthermore, step collapses do not occur and thus the total number of steps is preserved. The analysis becomes more involved for periodic 1D surface corrugations $[25,42]$ and radial geometries $[24,34]$ such as the one in the present study, where the facet height changes with time. For such geometries, boundary conditions consistent with step flow are in principle expected to involve microscale parameters, e.g., step collapse times, which result from solving discrete schemes for steps [24, 34]. Note, however, that there is a fundamental difference between the 1D periodic setting and radial geometries: in the former, steps on facets can be of opposite sign, which in turn may affect the nature of their interactions [25].

1.4. Notation and terminology. Writing $f=\mathcal{O}(g)$ implies that $f / g$ is bounded as a variable approaches a limit; similarly, $f \sim g$ means that $(f-g) / f$ approaches zero when a variable tends to a given limit. The symbols $\mathbb{N}, \mathbb{R}$, and $\left(x_{i}\right)_{i \in I}$ denote the set of natural and real numbers and a vector indexed by the set $I$, and $B^{d}(\mathbf{r}, \delta)$ is the open ball of radius $\delta>0$ centered at point $\mathbf{r}$ in the Euclidean space $\mathbb{R}^{d}$, where boldface symbols denote finite-dimensional vectors. The dot on top of a symbol denotes time derivative. The terms "continuum limit" and "macroscopic limit" are used interchangeably; ditto for "subgradient" and "extended gradient." We use the term "shock-like" loosely for solutions with a jump discontinuity not necessarily complying with the definition of a shock wave in hyperbolic laws. We use dimensional variables in sections 2, 4-5.3, and (partly) 7 , and we use nondimensional ones in sections 3 and $5.4-6.3$.

1.5. Article outline. The remainder of this article is organized as follows. In section 2, we describe the geometry and formulate the discrete equations of motion. In section 3, we give a proof for the existence of a unique solution to the ODE systems 


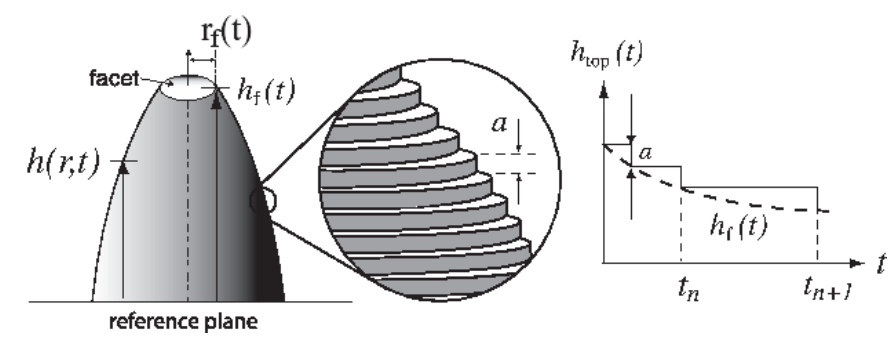

Fig. 2.1. Schematic of axisymmetric surface structure with a facet. At the macroscale, the height, $h(r, t)$, is continuous; the facet height and radius are $h_{\mathrm{f}}(t)$ and $r_{\mathrm{f}}(t)$. At the microscale, circular steps of atomic size a are evident so that the surface height is uniformly discretized; $t_{n}$ is the nth step collapse time, where $n$ enumerates steps in the initial configuration.

for M1 and M2 and thus prove the noncrossing property for steps. In section 4, we review the formal continuum limit away from the facet. In section 5 , starting with the subgradient theory, we introduce boundary conditions at the facet edge and describe explicit PDE solutions in the case without step interactions $\left(g_{3}=0\right)$. In section 6 , we numerically solve the free-boundary problems under self-similarity of the slope and compare continuum solutions to discrete simulations. In section 7 , we discuss an interpretation of the facet height as shock and discuss the convergence of the discrete schemes for $g_{3}=0$. In section 8 , we summarize our results and outline a few open problems. The appendices provide technical derivations needed in the main text.

2. Formulation. In this section we introduce the geometry of the problem and the governing equations (ODEs) of step motion. A key element of our modeling is the use of step mobilities that give rise to discrete dynamics prohibiting step crossing even for zero step interactions in M1 and M2.

2.1. Geometry. The geometry is shown in Figure 2.1. At the macroscale, the crystal surface is described by a continuous height profile, $h(r, t)$, with respect to a fixed $(x y-)$ plane of reference. The (circular) facet has zero-slope orientation, height $h_{\mathrm{f}}(t)$, and radius $r_{\mathrm{f}}(t)$. We expect that $\dot{h}_{\mathrm{f}}(t)$ is nonpositive and $\dot{r}_{\mathrm{f}}(t)$ is nonnegative, i.e., the facet loses height and expands, as in the surface diffusion case [33].

At the microscale, this configuration consists of concentric circular, descending steps of constant atomic height, $a$. The $i$ th step has radius $r_{i}(t)$, where initially (at $t=0) r_{i}$ is nonzero for $0 \leq i \leq N$ with $N \gg 1$; by convention, $r_{i}(t) \equiv 0$ if $i<0$ and $i>N$ for all $t \geq 0$. We take $N$ to be large yet finite, so that the structure can be considered as semi-infinite for all practical purposes (but not in sections 3.2 and 7.2). Steps are expected to shrink and collapse on top of the facet; only steps with $n \leq i \leq N$ are present at times $t_{n} \leq t<t_{n+1}$, where $t_{n}$ is the collapse time of the $n$th step of the initial configuration; by convention, set $r_{n}(t) \equiv 0$ if $t \geq t_{n}(n \geq 0)$. Thus, $i$ in nonzero $r_{i}$ is a variable index enumerating steps of the initial configuration that remain on the structure.

We assume that $r_{i+1}(0)>r_{i}(0)$ for all $i$; then, $r_{i+1}(t)>r_{i}(t)$ for all later times (see section 3). Accordingly, the discrete slopes defined by

$$
\mathcal{M}_{i}=\frac{a}{r_{i+1}-r_{i}}
$$

are positive $\left(\mathcal{M}_{i}>0\right)$ and bounded, $\mathcal{M}_{i} \leq \mathcal{O}(1)$. Near the top step, $r_{i+1}-r_{i}$ can be much larger than $a$ and, thus, $\mathcal{M}_{i}$ is small (as we verify numerically).

Let $h_{\text {top }}(t)$ denote the height of the top layer at time $t$ (see Figure 2.1). Because of step collapses on top of the facet, $h_{\text {top }}(t)$ must decay; evidently, $0<h_{\text {top }}-h_{\mathrm{f}}=\mathcal{O}(a) \downarrow$ 
0 in the macroscopic limit. The everywhere-continuous surface height, $h(r, t)$, is the continuum limit of the discrete (piecewise constant) height $h_{\mathrm{d}}(r, t)$ which satisfies

$$
h_{\text {top }}(t)-h_{\mathrm{d}}(r, t)=(i-n) a
$$

for $r_{i-1}<r<r_{i}$ and $t_{n-1} \leq t<t_{n}$. In the continuum limit, where $a \downarrow 0$ and $i a=\mathcal{O}(1)$, we assert that

$$
i a \rightarrow h_{\mathrm{f}}(0)-h(r, t) .
$$

The right-hand side is used as a continuum Lagrangian variable in section 7 .

2.2. Discrete equations of motion. Next, we address the specifics of M1 and M2. The (radial) velocity of the $i$ th step stems from (1.1) and (1.2) with $\mu^{0}=0$ :

$$
\dot{r}_{i}=-\nu \mathcal{G}_{i} \frac{r_{i}-r_{i-1}}{a} \mu_{i} ; \quad \mathcal{G}_{i}=\left\{\begin{array}{lc}
1 & \text { for M1 }, \\
\frac{r_{i}+r_{i-1}}{2 r_{i}} & \text { for M2 },
\end{array}\right.
$$

for $n \leq i \leq N$ and any $n \geq 0$, where $\nu$ has units of length/energy/time; recall that $r_{i-1}(t) \equiv 0$ if $i \leq n, t_{n-1} \leq t<t_{n}$. We claim that in M2 the above formula for $\mathcal{G}_{i}$ is compatible with the radial geometry; for a derivation of $\mathcal{G}_{i}$ from the BCF model with desorption and negative ES barrier, see Appendix A.

Note in passing that in the limit $a \downarrow 0$ with $r_{i}-r_{i-1}=\mathcal{O}(a), \mathcal{G}_{i} \rightarrow 1$ and ODEs (2.4) become $\partial_{t} h=-\nu \mu$, where $\mu$ is the macroscopic limit of $\mu_{i}$. This outcome is in agreement with Spohn's evaporation model [51]; see section 4 for details.

To determine $\mu_{i}$, we first describe the total step free energy, $E^{a}$, which accounts for step line tension as well as entropic and elastic-dipole step repulsive interactions:

$$
E^{a}(t)=\sum_{i=n}^{N} 2 \pi r_{i}(t)\left[g_{1} a+\mathcal{V}^{a}\left(r_{i}(t), r_{i+1}(t)\right)\right],
$$

where the pairwise interaction energy between steps of radii $r$ and $\rho$ is [24]

$$
2 \pi r \mathcal{V}^{a}(r, \rho):=2 \pi \check{g}_{3} \frac{r \rho}{\rho+r}\left(\frac{a}{\rho-r}\right)^{2} .
$$

In (2.5) and (2.6), $g_{1} a$ is the step line tension (energy/length) and $\check{g}_{3}$ expresses the strength of step-step repulsion per unit length of a step; for later algebraic convenience, replace $\check{g}_{3}$ by the (macroscopic) parameter [33]

$$
g_{3}=\frac{3}{2} \frac{\breve{g}_{3}}{a} .
$$

The step chemical potential is defined through the variational formula [35]

$$
\sum_{i} a \oint_{L_{i}} \mu_{i} v_{i} \mathrm{~d} s=\Omega \dot{E}^{a}(t)
$$

where $L_{i}$ is the $i$ th step curve and $v_{i}$ is the step velocity. Relation (2.8) implies

$$
\begin{aligned}
\mu_{i} & =\frac{\Omega}{a} \frac{1}{2 \pi r_{i}} \frac{\partial E^{a}}{\partial r_{i}} \\
& =\frac{\Omega g_{1}}{r_{i}}+\frac{\Omega}{r_{i} a} \frac{\partial\left\{r_{i}\left[\mathcal{V}^{a}\left(r_{i}, r_{i+1}\right)+\mathcal{V}^{a}\left(r_{i}, r_{i-1}\right)\right]\right\}}{\partial r_{i}} \\
& =\frac{\Omega g_{1}}{r_{i}}+\frac{2}{3} \Omega \frac{g_{3} a^{2}}{r_{i}}\left\{\psi\left(r_{i}, r_{i+1}\right)-\psi\left(r_{i-1}, r_{i}\right)+\frac{1}{r_{i}^{2}}\left[\phi\left(r_{i}, r_{i+1}\right)+\phi\left(r_{i-1}, r_{i}\right)\right]\right\}
\end{aligned}
$$

Copyright $@$ by SIAM. Unauthorized reproduction of this article is prohibited. 
where $\Omega$ is the atomic volume, $\Omega \approx a^{3}$, and

$$
\begin{aligned}
\psi(r, \rho) & =\frac{2 r \rho}{\rho+r} \frac{1}{(\rho-r)^{3}}, \\
\phi(r, \rho) & =\left(\frac{\rho r}{\rho+r}\right)^{2} \frac{1}{(\rho-r)^{2}} .
\end{aligned}
$$

Accordingly, we obtain the step velocity law

$$
\begin{aligned}
\dot{r}_{i}=-\frac{\Omega \nu g_{1}}{r_{i}} \frac{r_{i}-r_{i-1}}{a} \mathcal{G}_{i}\left\{1+\frac{2 g a^{2}}{3}[\right. & \psi\left(r_{i}, r_{i+1}\right)-\psi\left(r_{i-1}, r_{i}\right) \\
& \left.\left.+\frac{\phi\left(r_{i}, r_{i+1}\right)+\phi\left(r_{i-1}, r_{i}\right)}{r_{i}^{2}}\right]\right\}, \quad g=\frac{g_{3}}{g_{1}},
\end{aligned}
$$

where $n \leq i \leq N$ for $t_{n-1}<t<t_{n}$ and $r_{N+1}(t) \equiv 0 ; \mathcal{G}_{i}$ is defined in (2.4). The parameter $g$ is the relative strength of step line tension and step-step repulsion.

Equation (2.12) can be nondimensionalized by use of the variables $\tilde{r}_{i}=r_{i} / a$ and $\tilde{t}=\left(\nu g_{1} \Omega / a^{2}\right) t$ or, alternatively, via units with $a=1=\nu g_{1}$. We follow this route in sections 3 and 5.4-6.3. In sections $4-5.3$ and (partly) 7 , we retain the dimensional variables so as to indicate transparently the passage to the continuum limit.

3. Existence of unique discrete solution. In this section, we prove that, in contrast to diffusion limited (DL) kinetics [11], steps do not collide in M1 and M2 even for zero step interactions. We give separate proofs for $g=0$ and $g>0$. For $g=0$, our proof is uniform in the initial number, $N$, of steps, whereas this uniformity is lost for $g>0$. Thus, for $g=0$ we can consider a semi-infinite surface structure.

3.1. Case with $\boldsymbol{g}=\mathbf{0}$. The absence of step collisions can be loosely explained by inspection of (2.12) for $g=0$. Suppose two steps tend to coalesce at some time; then, the innermost step moves faster, whereas the other step is slowed down (because of the governing backward scheme), and step collision is thus avoided. Note that the assumed backward scheme is deemed natural in our setting, given that the preferred direction of motion of each step (with a minus sign in (2.4)) is toward the origin.

The main result of this subsection is the following.

Theorem 3.1. Let $N \in \mathbb{N}$ be the initial number of steps and $I=\{0,1, \ldots, N\}$. Consider $\mathbf{r}(t)=\left(r_{0}(t), r_{1}(t), \ldots, r_{N}(t)\right)$ and $\mathbf{f}(\mathbf{r})=\left(f_{0}(\mathbf{r}), f_{1}(\mathbf{r}), \ldots, f_{N}(\mathbf{r})\right)$, where

$$
f_{i}(\mathbf{r})= \begin{cases}-\mathcal{G}_{i} \frac{r_{i}-r_{i-1}}{r_{i}}, & r_{i} \neq 0, \\ 0, & r_{i}=0,\end{cases}
$$

and $r_{-1}(t) \equiv 0$ in the definition of $f_{0}$; the factor $\mathcal{G}_{i}$ is defined in $(2.4)$.

Then, there exists a unique global solution to the initial value problem (IVP)

$$
\dot{\mathbf{r}}=\mathbf{f}(\mathbf{r}), \mathbf{r}(0)=\mathbf{r}^{i n}, \mathbf{r}^{i n} \in W=\left\{\mathbf{x} \in \mathbb{R}^{N} \mid 0<x_{0}<\cdots<x_{N}\right\}
$$

in the domain $\Omega=\left\{\mathbf{x}=\left(x_{i}\right)_{i \in I} \mid x_{i} \neq 0, i \in I\right\}$. Furthermore, this solution stays in $W$ for $t \in\left[0, t_{0}\right)$ and in $W_{i}=\left\{\mathbf{x} \in \mathbb{R}^{N} \mid 0=x_{0}=\cdots=x_{i}, x_{i+1}<\cdots<x_{N}\right\}$ for $t \in$ $\left[t_{i}, t_{i+1}\right)$, where $t_{i}$ is the time when $r_{i}$ reaches 0 ; here, $0<t_{0}<t_{1}<\cdots<t_{N}<\infty$. $r_{i}(t)$ is continuous for all $t$ and smooth on $\left(t_{j-1}, t_{j}\right)$ for $j=0, \ldots, N$ (here, take $\left.t_{-1}=0\right)$. 
Proof. It suffices to give a proof for M1, i.e., if $\mathcal{G}_{i}=1$ for all $i$. Because in M2 the factor $\mathcal{G}_{i}$ is bounded for all $i$, the proof for M2 is almost identical; hence, we omit it.

First, we prove the existence of a unique local solution to problem (3.2). Observe that each $f_{i}$ is smooth on $W$ with $\nabla f_{i}=\left(0, \ldots, 0, \frac{1}{r_{i}},-\frac{r_{i-1}}{r_{i}^{2}}, 0, \ldots, 0\right)$. So, for every $\mathbf{r} \in W$, let $0<\delta<\min _{i \in I}\left\{r_{i}\right\}$. Then, for any $\mathbf{y} \in B^{N}(\mathbf{r}, \delta)$ we have

$$
\left|\nabla f_{i}(\mathbf{y})\right|^{2}=\frac{1}{y_{i}^{2}}+\frac{y_{i-1}^{2}}{y_{i}^{4}} \leq\left(\frac{1}{r_{i}-\delta}\right)^{2}+\frac{\left(r_{i-1}+\delta\right)^{2}}{\left(r_{i+1}-\delta\right)^{4}}<\infty
$$

for each $i$. Hence, $\mathbf{f}$ is locally Lipschitz; by the Picard-Lindelöf theorem [19], IVP (3.2) has a unique local solution in $\Omega$. This local solution is smooth since $\mathbf{f}$ is smooth.

Let $\mathbf{r}^{i n} \in W$ and suppose that $[0, T)$ is a maximal interval on which the problem $\dot{\mathbf{r}}=\mathbf{f}(\mathbf{r}), \mathbf{r}(0)=\mathbf{r}^{i n}$ has a solution in $W$. Since $r_{0}(t)=r_{0}^{i n}-t$, we establish an upper bound for $T$ :

$$
T \leq r_{0}^{i n}<\infty
$$

We will show that $\mathbf{r}(t)$ approaches $\partial W$ as $t \uparrow T$. Suppose by contradiction that $\mathbf{r}(t)$ does not approach $\partial W$ as $t \uparrow T$. Then, in particular, we have $\min _{i} \inf _{t \in[0, T)} r_{i}(t)>0$ and $\mathbf{f}$ is uniformly bounded on the image $\mathbf{r}([0, T))$. Thus, $\mathbf{r}(t)$ is Cauchy-continuous. Therefore, the (classical) solution can be extended to $[0, T+b)$ by a standard theorem in the theory of ODE [7] that states that an extension of the solution exists at $T$ if $\lim _{t \uparrow T} \mathbf{r}(t)$ exists in $W$. This assertion contradicts the maximality of $T$. We thus conclude that $\mathbf{r}(t)$ approaches $\partial W$ as $t \uparrow T$.

Now, partition $\partial W$ into $V_{1}=\left\{\mathbf{x} \in \mathbb{R}^{N} \mid \exists i \in I \backslash\{0\}\right.$ such that $\left.x_{i}=x_{i-1}\right\}$ and $V_{2}=\partial W \backslash V_{1}$. Suppose by contradiction that $\mathbf{r}(T) \in V_{1}$. Let $\left\{r_{j}\right\}_{j \in J}$ be a set consisting of all components of $\mathbf{r}$ such that $r_{j}(T)=r_{j-1}(T)$. In particular, $J$ is not empty. So let $j_{0} \in J$ be the smallest index. Then, given any $\epsilon>0$, there exists some interval $(T-c, T)$ on which $\dot{r}_{j_{0}}=-\frac{r_{j_{0}}-r_{j_{0}-1}}{r_{j_{0}}}>-\epsilon$. By taking $\epsilon=\sup _{[0, T)}\left|\dot{r}_{j_{0}-1}\right|$, we see that $\frac{d}{d t}\left(r_{j_{0}}-r_{j_{0}-1}\right)=\dot{r}_{j_{0}}-\dot{r}_{j_{0}-1}>-\epsilon+\epsilon=0$ on $(T-c, T)$. Hence, $\lim _{t \uparrow T}\left[r_{j_{0}}(t)-r_{j_{0}-1}(t)\right] \neq 0$ and $j_{0}$ cannot be in $J$. By this contradiction, we conclude that (3.2) has a unique global solution whose trajectory meets the subset $\left\{r_{0}=0\right\} \backslash V_{1}$ of a hyperplane $\left\{r_{0}=0\right\}$ at some finite time $T$. Define $t_{0}:=T$; by the definition of $f_{0}, r_{0}(t) \equiv 0$ for $t \in\left[t_{0}, \infty\right)$. Next, proceed as in the above argument with the dimension of the solution reduced by 1 . Repeat this procedure until $\mathbf{r}=0$.

Remark 3.2. In the above proof, $t_{n}$ are the step collapse times. For $g=0$ and conical initial data (i.e., $r_{i}(0)$ linear with $i$ ), we will obtain explicit solutions for the top two steps of ODEs (3.2) with indices $i=n, n+1$, and $t \in\left(t_{n-1}, t_{n}\right)$. For this special case, the explicit solution indicates that steps do not collide, as verified through our numerics (section 6).

Remark 3.3. A consequence of Theorem 3.1 is that the discrete slope $\mathcal{M}_{i}$ is positive and bounded for any $i$ and $N$; the step mobility $\nu_{i}$ is also positive.

Remark 3.4. The proof of Theorem 3.1 holds as $N \rightarrow \infty$ (for semi-infinite structures).

Corollary 3.5. If the continuum limit of the solution to IVP (3.2) exists, this limit yields a monotone continuum-scale height for all time $t>0$ provided the height profile is strictly monotone at $t=0$.

In fact, we will verify the last statement for the case of conical initial data through an exact closed-form solution of the evolution PDE for the slope (see section 5.4). 
3.2. Case with $\boldsymbol{g}>\mathbf{0}$. For positive $g$, the proof for the existence of a unique solution to ODE system (2.12) and the noncrossing property of steps for such a solution is in the spirit of the proof for $g=0$. However, for $g>0, \dot{\mathbf{r}}(t)$ becomes unbounded as $r_{j} \rightarrow r_{j-1}$ for some $j$ in the step interaction terms of $\mu_{i}$. Hence, the proof that we provide below holds only when $N$ is finite.

The core result of this subsection is the following.

TheOREM 3.6. Let $N \in \mathbb{N}$ be the initial number of steps and $I=\{0,1, \ldots, N\}$. Consider $\mathbf{r}(t)=\left(r_{0}(t), r_{1}(t), \ldots, r_{N}(t)\right)$ and $\mathbf{f}(\mathbf{r})=\left(f_{0}(\mathbf{r}), f_{1}(\mathbf{r}), \ldots, f_{N}(\mathbf{r})\right)$, where

$$
\begin{aligned}
f_{i}(\mathbf{r})=-\mathcal{G}_{i} \frac{r_{i}-r_{i-1}}{r_{i}}\left\{1+\frac{2 g}{3}[\right. & \psi\left(r_{i}, r_{i+1}\right)-\psi\left(r_{i-1}, r_{i}\right) \\
& \left.\left.+\frac{\phi\left(r_{i}, r_{i+1}\right)+\phi\left(r_{i-1}, r_{i}\right)}{r_{i}^{2}}\right]\right\} \quad \text { if } r_{i} \neq 0,
\end{aligned}
$$

$$
f_{i}(\mathbf{r})=0 \quad \text { if } r_{i}=0 .
$$

Here, $\psi$ and $\phi$ are defined by (2.10) and (2.11), respectively; $r_{-1}(t) \equiv 0$ in the definition of $f_{0}$ while $r_{N+1}(t) \equiv 0$ in the definition of $f_{N}$; and $\mathcal{G}_{i}$ is defined in (2.4).

Then, there exists a unique global solution to the IVP

$$
\dot{\mathbf{r}}=f(\mathbf{r}), \mathbf{r}(0)=\mathbf{r}^{i n}, \mathbf{r}^{i n} \in W=\left\{\mathbf{x} \in \mathbb{R}^{N} \mid 0<x_{0}<\cdots<x_{N}\right\}
$$

in the domain $\Omega=\left\{\mathbf{x}=\left(x_{i}\right)_{i \in I} \mid x_{i} \neq 0, i \in I\right\}$. Furthermore, this solution stays in $W$ for $t \in\left[0, t_{0}\right)$ and in $W_{i}=\left\{\mathbf{x} \in \mathbb{R}^{N} \mid 0=x_{0}=\cdots=x_{i}, x_{i+1}<\cdots<x_{N}\right\}$ for $t \in$ $\left[t_{i}, t_{i+1}\right)$, where $t_{i}$ is the time when $r_{i}$ reaches 0 . Here, $0<t_{0}<t_{1}<\cdots<t_{N}<\infty$; and $r_{i}(t)$ is continuous for all $t$ and is smooth on $\left(t_{j-1}, t_{j}\right)$ for $j=0, \ldots, N$.

Proof. Again, we provide a proof only for M1, where $\mathcal{G}_{i}=1$; the proof for M2 is very similar and thus omitted.

In the spirit of the proof for $g=0$, we first prove the existence of a unique local solution to problem (3.6). Observe that each $f_{i}$ is smooth on $W$. For each vector $\mathbf{r} \in W$, let $0<\delta<\min _{i \in I}\left\{r_{i},\left(r_{i}-r_{i-1}\right) / 4\right\}$. Then, by recourse to ODEs (2.12), for all $\mathbf{y} \in B^{N}(\mathbf{r}, \delta)$ we note the following bound in regard to partial derivatives of $f_{i}$ :

$$
\begin{aligned}
& \left|\frac{\partial}{\partial y_{i}}\left(\frac{y_{i}-y_{i-1}}{y_{i}} \psi\left(y_{i}, y_{i+1}\right)\right)\right|^{2} \\
& =\mid \frac{2 y_{i} y_{i+1}}{y_{i}\left(y_{i}+y_{i+1}\right)} \frac{1}{\left(y_{i+1}-y_{i}\right)^{3}}-\left(1-\frac{y_{i-1}}{y_{i}}\right) \frac{2 y_{i} y_{i+1}}{\left(y_{i}+y_{i+1}\right)^{2}} \frac{1}{\left(y_{i+1}-y_{i}\right)^{3}} \\
& \quad+\left.3\left(1-\frac{y_{i-1}}{y_{i}}\right) \frac{2 y_{i} y_{i+1}}{y_{i}+y_{i+1}} \frac{1}{\left(y_{i+1}-y_{i}\right)^{4}}\right|^{2} \leq K(\delta),
\end{aligned}
$$

where $K(\delta)=\mathcal{O}\left(1 / \delta^{3}\right)$ for small $\delta$. In (3.7), we used an inverse triangle inequality, $\left|y_{i}-y_{i-1}\right| \geq\left|r_{i}-r_{i-1}\right|-\left|y_{i}-r_{i}\right|-\left|y_{i+1}-r_{i+1}\right| \geq 2 \delta$, in order to obtain a bound for each term; for example, the first term in the right-hand side is bounded by $\frac{\left(r_{i}+\delta\right)\left(r_{i+1}+\delta\right)}{\left(r_{i}-\delta\right)\left(r_{i}+r_{i+1}-2 \delta\right)} \frac{1}{4 \delta^{3}}$. We omit the details on the rest of partial derivatives for $f_{i}$, since the procedure is similar to the one for (3.7). Thus, every $\left|\nabla f_{i}\right|$ is bounded from above. Therefore, $\mathbf{f}$ is locally Lipschitz; by the Picard-Lindelöf theorem [19], we assert the existence of a unique local solution to problem (3.6).

Now, as before, let $\mathbf{r}^{i n} \in W$ and $[0, T)$ be a maximal interval on which the problem

$$
\dot{\mathbf{r}}=\mathbf{f}(\mathbf{r}), \quad \mathbf{r}(0)=\mathbf{r}^{i n}
$$

Copyright (c) by SIAM. Unauthorized reproduction of this article is prohibited. 
has a (classical) solution in $W$. Notice that on $W, \dot{r}_{0}(t)<\dot{r}_{0}^{(0)}(t)$, where $r_{0}^{(0)}(t)$ denotes the first component of the solution for IVP (3.2) with (3.1). Thus, $r_{0}(t)<$ $r_{0}^{(0)}(t)=r_{0}^{i n}-t$ and we obtain an upper bound for $T$ :

$$
T \leq r_{0}^{i n}<\infty .
$$

By neglecting the negative terms in (3.5) for each $i$, we obtain the inequality

$$
\dot{r}_{i}(t) \leq \frac{4 g}{3} \frac{1}{\left(r_{i}-r_{i-1}\right)^{2}} .
$$

We now show that $\mathbf{r}(t)$ approaches $\partial W$ as $t \uparrow T$. Suppose by contradiction that this statement is false. Then, $r_{i} \nrightarrow r_{i-1}$ and the right-hand side of (3.10) is clearly bounded above on $[0, T)$. Hence, $\mathbf{f}(\mathbf{r})$ is uniformly bounded on the image $\mathbf{r}([0, T))$ and, by recourse to the argument given in the proof for Theorem 3.1, we conclude that $\mathbf{r}(t)$ approaches $\partial W$ as $t \uparrow T$.

As in the proof of Theorem 3.1, now partition $\partial W$ into $V_{1}$ and $V_{2}=\partial W \backslash V_{1}$. Next, we prove that $\mathbf{r}(t) \rightarrow V_{2}$ as $t \rightarrow T$. Let $\left\{r_{j}\right\}_{j \in J}$ be a set consisting components of $\mathbf{r}$ such that $r_{j}(T)=r_{j-1}(T)$. Suppose by contradiction that $\mathbf{r}$ reaches $V_{1}$ at time $T$; this implies that the set $J$ is nonempty. If for some $j \in J, \dot{r}_{j}$ grows unbounded in the positive direction near time $T$ yet $\dot{r}_{j-1}$ is bounded above, then $\dot{r}_{j}-\dot{r}_{j-1}>0$ on some interval $(T-c, T)$, which contradicts the definitions of $T$ and $J$. Thus, the desired result follows: $\mathbf{r}$ does not reach $V_{1}$ by time $T$.

Because $J$ is nonempty, there exists the smallest index $j_{0} \in J$ and the largest index $j_{*} \in J$ such that the sequence $j_{0}, j_{0}+1, \ldots, j_{*}-1, j_{*}$ is contained in $J$. Since $j_{0}-1 \notin J$, we have that $r_{j_{0}-1}-r_{j_{0}-2} \nrightarrow 0$ and $\psi\left(r_{j_{0}-2}, r_{j_{0}-1}\right)$ is bounded. Thus, the only positive term in $f_{j_{0}-1}(\mathbf{r})$ is bounded near time $T$, so $\dot{r}_{j_{0}-1}=f_{j_{0}-1}(\mathbf{r})$ is bounded from above in some interval $(T-\tilde{c}, T)$. On the other hand, $j_{*}+1 \notin J$ implies that $\psi\left(r_{j_{*}}, r_{j_{*}+1}\right), \phi\left(r_{j_{*}}, r_{j_{*}+1}\right)$ are both bounded. Also, since $j_{*} \in J$, we deduce that $\phi\left(r_{j_{*}-1}, r_{j_{*}}\right)$ grows as $\left(r_{j_{*}}-r_{j_{*}-1}\right)^{-2}$, whereas $\psi\left(r_{j_{*}-1}, r_{j_{*}}\right)$ grows as $\left(r_{j_{*}}-r_{j_{*}-1}\right)^{-3}$. Thus, $\dot{r}_{j_{*}}(t)$ must grow unbounded in the positive direction as $t \rightarrow T$.

Hence, the properties that $r_{j_{0}-1}$ is bounded and $r_{j_{*}}$ is unbounded warrant that there exists some $j \in J$ for which $\dot{r}_{j}$ grows unbounded above for times near $T$ yet $\dot{r}_{j-1}$ is bounded above. We conclude that IVP (3.6) has a unique global solution $\mathbf{r}(t)$ that ends when the trajectory meets the subset $\left\{r_{0}=0\right\} \backslash V_{1}$ of the hyperplane $\left\{r_{0}=0\right\}$ at some finite time $T$. Define $t_{0}:=T$; by definition (3.5) of $f_{0}, r_{0}(t) \equiv 0$ for $t \in\left[t_{0}, \infty\right)$. Now proceed as above with a new IVP in which the dimension of solution is reduced by 1 . Repeat this procedure until $\mathbf{r}=0$.

Remark 3.7. The above proof relies on the fact that $J$ is a finite set and there exists the largest index in $J$ (i.e., $J$ is bounded); in particular, it is necessary that $N$ be finite. Thus, we may not use our proof for $g>0$ in order to assert positivity of the discrete slope for a semi-infinite structure (as $N \rightarrow \infty$ ).

Remark 3.8. For $g>0$, one can state a result analogous to Corollary 3.5.

4. Continuum limit away from facet. In this section, we derive Spohn's PDE [51] for the surface height outside the facet from step velocity law (2.12). Our computations are formal, invoking notions of pointwise convergence (with the exception of (4.6) for $\mu$ ); similar, yet more detailed, heuristic derivations are presented in $[33,35]$ as parts of the (technically more involved) case with surface diffusion, where the step velocity is the difference of adatom fluxes each of which is expressed in terms of differences of step chemical potentials at neighboring steps. We emphasize that the 
derived continuum laws are valid for $r>r_{\mathrm{f}}(t)$. The facet position, $r_{\mathrm{f}}(t)$, should be determined as part of the solution of a free-boundary problem (see section 5 ).

Consider steps $i$ with $1 \ll n \ll i \ll N$ and $i a=\mathcal{O}(1)$ in view of (2.3). We assume that the discrete slopes, $\mathcal{M}_{i}$, are fixed; cf. (2.1). On each terrace, $r_{i-1}<r<r_{i}$, we have $h_{\mathrm{d}}(r, t)=$ const. For the continuum-scale height function $h(r, t)$, slope $m(r, t)$, and chemical potential $\mu(r, t)$ to make sense from limits of $a i, \mathcal{M}_{i}$, and $\mu_{i}$, respectively, we assume that the terrace width varies slowly in $i$ and $r_{i+1}-r_{i} \ll r_{i}, r_{i+1}$. When these conditions are not met, discrete effects are expected to become important.

As $r \uparrow r_{i}(t)$, differentiation of $h_{\mathrm{d}}(r, t)$ with respect to time yields [35]

$$
\dot{r}_{i} \rightarrow \partial_{t} h(r, t) /\left.m(r, t)\right|_{r=r_{i}(t)} \quad \text { as } a \rightarrow 0,
$$

where $m(r, t)=-\partial_{r} h(r, t)$ and $r>r_{\mathrm{f}}(t)$. Equation (4.1) unveils the limit of the right-hand side of (2.12).

On the other hand, the discrete mobility, $\nu_{i}=\nu \mathcal{G}_{i}\left(r_{i}-r_{i-1}\right) / a$, approaches

$$
\nu_{i} \rightarrow \nu /\left.m(r, t)\right|_{r=r_{i}(t)}, \quad r>r_{\mathrm{f}}(t),
$$

for both M1 and M2 (since $\mathcal{G}_{i} \rightarrow 1$ ). Thus, if $\mu_{i}(t) \rightarrow \mu(r, t),(4.1)$ and (4.2) yield

$$
\partial_{t} h(r, t)=-\nu \mu(r, t), \quad r>r_{\mathrm{f}}(t) .
$$

There are at least two routes to obtaining a formula for $\mu$. One way is to directly take the limit of (2.9) under the condition $\mathcal{O}(a)=r_{i}-r_{i-1} \ll r_{i}$ for large $i$. For this purpose, the right-hand side of (2.9) is expressed in terms of discrete slopes, $\mathcal{M}_{i}$, with the main substitution

$$
r_{i \pm 1} \sim r \pm \frac{a}{m(r, t)}, \quad r=r_{i}
$$

The algebraic manipulations are detailed in [33]. As a result, we obtain

$$
\mu(r, t)=\frac{\Omega g_{1}}{r}+\Omega g_{3} \frac{1}{r} \frac{\partial}{\partial r}\left(r m^{2}\right), \quad r>r_{\mathrm{f}}(t) .
$$

Alternatively, by (2.5) and (2.8) one can write $\mu(r, t)$ as the first variation of the continuum limit of $E^{a}$. In view of the coarea formula $\sum_{i} a \oint_{L_{i}} \cdot d s \rightarrow \int|\nabla h| \cdot d A$, where $L_{i}$ denotes a step curve [35], the limit of $E^{a}$ is the known surface free energy [18]

$$
E^{a} \underset{a \rightarrow 0}{\longrightarrow} E(h)=\iint \gamma(\nabla h) \mathrm{d} A, \quad \gamma(\nabla h)=\left(g_{1}|\nabla h|+\frac{g_{3}}{3}|\nabla h|^{3}\right),
$$

where $d A=\mathrm{d} x \mathrm{~d} y$. Now, $\mu$ is obtained through [33, 35]

$$
\left\langle\mu, \partial_{t} h\right\rangle=\Omega \dot{E} \Rightarrow \mu(r, t) \equiv \Omega \frac{\delta E}{\delta h}, \quad r>r_{\mathrm{f}}(t),
$$

where $\langle\mu, \varphi\rangle=\iint \mu(x, y) \varphi(x, y) \mathrm{d} A$ denotes the $L^{2}$-inner product, and $\delta E / \delta h$ is the variational derivative of $E(h)$. Equation (4.6) is consistent with continuum thermodynamics and is valid outside the facet. The computation of $\delta E / \delta h$ leads to (4.4).

Equations (4.3) and (4.4) yield a PDE for the height, $h(r, t)$,

$$
\partial_{t} h=-\nu \Omega g_{1} \operatorname{div} \boldsymbol{\xi}, \quad r>r_{\mathrm{f}}(t),
$$


where

$$
\xi=\xi e_{r}, \quad \xi(r, t)=1+g m(r, t)^{2}, \quad r>r_{\mathrm{f}}(t),
$$

and $e_{r}$ is the unit radial vector. The PDE for the positive slope, $m=-\partial_{r} h$, reads

$$
\partial_{t} m=-\nu \Omega g_{1}\left\{r^{-2}-g \partial_{r}\left[r^{-1} \partial_{r}\left(r m^{2}\right)\right]\right\}, \quad r>r_{\mathrm{f}}(t) .
$$

Note that (4.7) has a mass conservation form, where $\boldsymbol{\xi}$ is a vector-valued flux associated with the flow $\partial_{t} h$ (and $h$ plays the role of a "density").

We emphasize that both M1 and M2 reduce to the same (parabolic) PDE (4.7) outside the facet. However, as we discuss in section 5 , the facet boundary conditions for (4.7) must be adjusted in correspondence to the particulars of M1 or M2 so that the continuum slope is in agreement with step simulations in each case.

5. Subgradient formalism and free-boundary conditions. In this section, we start with the subgradient formalism in order to extract the natural boundary conditions at the facet edge; these conditions form our starting point for the comparison of continuum solutions to step simulations. Our numerical simulations (section 6) indicate that the natural boundary conditions yield a continuum slope in agreement with predictions of M1 but not M2. In anticipation of this discrepancy, in section 5.2 we propose a set of modified boundary conditions by incorporating the discrete geometric factor on top of the facet into one of the conditions in terms of a jump of the flux. (See section 6 for numerical studies of this replacement.) In section 5.3, we prescribe linear initial data. In section 5.4, we analytically solve the free-boundary problems for $g=0$ (noninteracting steps) to single out differences between the two continuum solutions, without and with a flux jump.

5.1. Subgradient formalism. A guiding principle in the analysis of PDE (4.7) with (4.8) is that the height profile evolves by the most rapid decrease of the free energy, $E(h)$. Qualitatively speaking, this principle is consistent with step motion law (2.4) in view of (2.9); clearly, the trajectory of $\left(r_{0}(t), r_{1}(t), \ldots, r_{N}(t)\right)$ is driven by the decrease of the total step free energy, $E^{a}(t)$. At the moment, we lack a more precise energy-based connection of the subgradient theory with steps. ${ }^{3}$

By the extended gradient formalism, the evolution law for $h$ is stated as [27]

$$
\partial_{t} h(r, t)=-\nu \Omega g_{1} \operatorname{div} \boldsymbol{\xi}(r, t) \quad \text { for all } r \geq 0,
$$

where $\operatorname{div} \boldsymbol{\xi}$ is proportional to $\delta E / \delta h$ outside the facet, $|\boldsymbol{\xi}| \leq 1$ on the facet, and $\boldsymbol{\xi}=$ $\xi e_{r}$. The flux $\boldsymbol{\xi}$ is uniquely determined from (5.1) under the assumption of sufficient regularity. Physically, (5.1) expresses global mass conservation for the height, $h$, if $\boldsymbol{\xi}$ is interpreted as a mass flux comprising atoms exchanged with the vapor. Some elements of the subgradient theory are reviewed in Appendix B.

5.1.1. Natural boundary conditions. Next, we write explicit boundary conditions for (4.7) with (4.8). Note that four (three) conditions are needed, since the PDE for $h$ is of second (first) order for $g>0(g=0)$, and $r_{\mathrm{f}}(t)$ and $h_{\mathrm{f}}(t)$ are parts of the solution. If $r<r_{\mathrm{f}}(t)$, then $h=h_{\mathrm{f}}(t)$. By continuity of the height, $h(\cdot, t)$, we write

$$
h_{\mathrm{f}}(t)=h(r, t) \quad \text { as } r \downarrow r_{\mathrm{f}}(t) .
$$

\footnotetext{
${ }^{3}$ To the best of our knowledge, so far such a connection has been established rigorously for a $1 \mathrm{D}$ finite step train connecting semi-infinite facets in ADL kinetics [1, 2].
} 
This condition is natural on physical grounds but is also inferred from the functional space for solutions to (5.1) (see Appendix B). Equation (5.1) on the facet reads $\dot{h}_{f}=-\nu \Omega g_{1} r^{-1} \partial_{r}(r \xi)$, by which $\nu \Omega g_{1} \xi(r, t)=-(r / 2) \dot{h}_{\mathrm{f}}+C(t) / r$ if $r<r_{\mathrm{f}}(t)$; set $C(t) \equiv 0$ so that $\xi(\cdot, t)$ is bounded. Thus, continuity of $\xi(\cdot, t)$ entails

$$
-\frac{r_{\mathrm{f}}(t)}{2} \dot{h}_{f}=\nu \Omega g_{1}\left[1+g m(r, t)^{2}\right] \quad \text { as } r \downarrow r_{\mathrm{f}}(t) .
$$

For large $r$, the solution must be compatible with the prescribed initial data:

$$
h(r, t) \sim h(r, 0) \text {; or } m(r, t) \sim-\partial_{r} h(r, 0) \quad \text { as } r \rightarrow \infty,
$$

which is a "far field" condition.

For $g>0$, one more condition must be imposed. Recall that $|\xi| \leq 1$ on the facet, $\xi=1+g m^{2}>1$ outside the facet, and $\xi$ is continuous. Thus, the slope is continuous:

$$
\lim _{r \downarrow r_{\mathrm{f}}(t)} m(r, t)=0 \text {. }
$$

Equations (5.2)-(5.5) form the set of natural boundary conditions for PDE (4.7) outside the facet. In view of (5.5), the differentiation of (5.2) with respect to $t$ can be used to replace (5.2) and (5.3) by a single condition via elimination of $\dot{h}_{\mathrm{f}}$.

5.2. Alternate condition: Flux jump at facet edge. Next, we modify condition (5.3) by introducing an ad hoc jump of the flux $\boldsymbol{\xi}(\cdot, t)$. Our goal is to describe the effect on the macroscopic limit of individual steps collapsing on top of the facet by retaining the variables (height, slope, flux) of the natural boundary conditions.

Physically, the main idea can be outlined as follows. In the continuum limit, $t_{n}$ is treated as the continuous time, $t[34]$. A reasonable boundary condition that captures the events of collapsing top layers must express via some effective averaging in time the mass exchange between top (annihilated) steps and vapor in the time intervals $\left[t_{n}, t_{n+1}\right]$. We propose an ad hoc possible remedy for the underlying averaging process: a, in principle time-dependent, jump of the flux $\boldsymbol{\xi}(\cdot, t)$. In the case with self-similarity of slopes, which we focus on, this jump is expressed by a time-independent factor due to a scaling property of $t_{n}$ with large $n$. More generally, it is hoped that this approach can be applied to full time-dependent settings without restriction to self-similarity.

For the step dynamics of M2, in particular, we specify the jump in terms of values of $\mathcal{G}_{i}(t)$ for pairs of extremal steps at $t=t_{n}$. This factor encapsulates, in a simple geometric form, information about the facet microstructure. At the moment, this choice is essentially empirical (see Remarks 5.2 and 5.3): the only firm justification that we can provide is the observed agreement of the ensuing continuum slope with step simulations for M2 (section 6).

Our proposal is to keep evolution law (5.1) for $0 \leq r<r_{\mathrm{f}}(t)$ and $r>r_{\mathrm{f}}(t)$, along with height continuity and (for $g>0$ ) slope continuity, but replace (5.3) by the generalized condition

$$
-\frac{r_{\mathrm{f}}(t)}{2} \dot{h}_{f}=\nu \Omega g_{1} \widetilde{\mathcal{G}}(t ; g)\left[1+g m(r, t)^{2}\right] \quad \text { as } r \downarrow r_{\mathrm{f}}(t),
$$

where $\widetilde{\mathcal{G}}(t ; g)$ is allowed to differ from unity; we henceforth suppress the $g$-dependence of $\widetilde{\mathcal{G}}$. The function $\widetilde{\mathcal{G}}(t)$ should be compatible with the vanishing of individual, atomic layers on top of the facet. In particular, for M2 we propose the definition

$$
\left.\widetilde{\mathcal{G}}(t) \equiv \mathcal{G}_{i}\left(t_{n}\right)\right|_{i=n+2}=\frac{r_{n+2}\left(t_{n}\right)+r_{n+1}\left(t_{n}\right)}{2 r_{n+2}\left(t_{n}\right)}
$$

for $t_{n} \leq t<t_{n+1}$, accounting for the two extremal steps; recall that $r_{n}\left(t_{n}\right)=0$. 
Remark 5.1. In section 6.2, we numerically test (5.6) and (5.7). First, for M1 we show that for large $t$, the replacement of $\widetilde{\mathcal{G}}(t)$ by unity produces continuum slopes in excellent agreement with our step simulations. For M2, we demonstrate that if $n$ (and $t$ ) is large, (i) (5.7) yields $\widetilde{\mathcal{G}}(t ; g) \sim \mathfrak{c}(g)$, where $\mathfrak{c}$ is a constant computed from data for $t_{n}$; and (ii) the resulting continuum slopes closely follow step simulations. In the subgradient formalism, $\mathfrak{c}=1$.

Remark 5.2. Notably, some other choices which are almost as simple as (5.7) fail to yield equally satisfying numerical results for $g>0$. For example, suppose we set $\widetilde{\mathcal{G}}(t) \equiv \mathcal{G}_{n+2+k}\left(t_{n}\right)$ for $k+1 \in \mathbb{N}$ and $t_{n} \leq t<t_{n+1}$, viewing the jump as a function of $k$ for every fixed $n$. We numerically observe that the value $k=0$ is best for agreement of the continuum prediction with step simulations for M2; in fact, the factor $\widetilde{\mathcal{G}}$ is found to be monotonically increasing with $k$ in this scenario.

Remark 5.3. Especially for noninteracting steps, $g=0$, we can claim that the continuum theory consistent with M2 has a flux jump yet does not require input from discrete simulations. Suppose we employ (5.7) in this case; then, we find that $\widetilde{\mathcal{G}}(t)$ becomes approximately equal to 0.766 for large $n$. See Appendix $\mathrm{C}$ for related computations. This value produces a continuum slope in agreement with discrete simulations (see section 6.1.2). On the other hand, by interpreting the facet height as shock, we can evaluate the flux jump from a Rankine-Hugoniot condition; then, we find $\widetilde{\mathcal{G}}(t)=3 / 4$ (section 7.1), which also yields a continuum slope in excellent agreement with step simulations (section 6.1.2). This observation indicates the empirical nature of (5.7). A detailed discussion on the shock picture is provided in section 7 .

An emerging question is whether our generalized boundary condition (5.6) for the flux should require input from discrete simulation data, as suggested by (5.7), or, in a more appealing scenario, it can be expressed within a full ( $t_{n}$-independent) continuum framework for $g>0$. This issue is the subject of current research.

5.3. Initial data. In the remainder of this article, we consider an initial conical profile of unit slope. Hence, we impose the initial height profile

$$
h(r, 0) \equiv h_{0}(r)= \begin{cases}h_{\mathrm{f} 0}, & r<r_{\mathrm{f} 0}, \\ h_{\mathrm{f} 0}-\left(r-r_{\mathrm{f} 0}\right), & r>r_{\mathrm{f} 0},\end{cases}
$$

where $r_{\mathrm{f} 0}=r_{\mathrm{f}}(0)$ and $h_{\mathrm{f} 0}=h_{\mathrm{f}}(0)$. This profile corresponds to the initial step train

$$
r_{i}(0)=r_{\mathrm{f} 0}+i a, \quad i=0,1, \ldots, N,
$$

where the top layer is located at height $h_{\mathrm{fo}}$.

5.4. Exactly solved case: Zero step interaction $(g=0)$. In this subsection, we analytically solve the free-boundary problems of sections 5.1 and 5.2 in the absence of step-step interaction. In this case, the continuity of slope, condition (5.5), is not applicable. First, we restrict attention to a continuous flux for $\widetilde{\mathcal{G}}(t)=1$; the resulting formula for $m(r, t)$ exhibits self-similar behavior. Second, we extend our computation to $\widetilde{\mathcal{G}}(t) \neq 1$. Detailed derivations are presented in Appendix D. In the remainder of this section, we employ units with $\nu \Omega g_{1}=1=a$.

For $g=0$, PDE (4.7) reduces to

$$
\partial_{t} h=-\frac{1}{r}, \quad r>r_{\mathrm{f}}(t),
$$

which leads to the solution

$$
h(r, t)=h_{0}(r)-\frac{t}{r}, \quad r>r_{\mathrm{f}}(t) ; \quad h(r, t)=h_{\mathrm{f}}(t), \quad r<r_{\mathrm{f}}(t) .
$$


Hence, the slope profile is

$$
m(r, t)=1-\frac{t}{r^{2}}, \quad r>r_{\mathrm{f}}(t) ; \quad m(r, t)=0, \quad r<r_{\mathrm{f}}(t) .
$$

It remains to compute the facet radius, $r_{\mathrm{f}}(t)$, by applying initial data (5.8). The height continuity, $h_{\mathrm{f}}(t)=h_{\mathrm{f} 0}+r_{\mathrm{f} 0}-r_{\mathrm{f}}-t / r_{\mathrm{f}}$ along with generalized condition (5.6) on $\xi(\cdot, t)$ yield an ODE for $r_{\mathrm{f}}(t)$. We now distinguish two cases for the flux $\boldsymbol{\xi}$.

5.4.1. Continuous flux $\boldsymbol{\xi}$. In the special case without flux jump, $\widetilde{\mathcal{G}}(t) \equiv 1$, $r_{\mathrm{f}}(t)$ is computed in simple closed form for all $t \geq 0$ (see Appendix D); in particular,

$$
r_{\mathrm{f}}(t) \sim \sqrt{3 t} \quad \text { as } t \rightarrow \infty .
$$

Accordingly, we compute the facet height:

$$
h_{\mathrm{f}}(t) \sim h_{\mathrm{f} 0}+r_{\mathrm{f} 0}-\frac{4}{\sqrt{3}} \sqrt{t} \quad \text { as } t \rightarrow \infty .
$$

The slope has the form $m(r, t)=\mathfrak{m}(\eta)$ with $\eta=r / \sqrt{t}$, where $\eta \sim$ const at the facet edge for large $t$. By (5.12) and (5.13), note that

$$
m(r, t) \rightarrow m_{\mathrm{f}}:=\mathfrak{m}(\sqrt{3})=2 / 3 \quad \text { as } r \downarrow r_{\mathrm{f}}(t), t \rightarrow \infty .
$$

5.4.2. Discontinuous flux $\boldsymbol{\xi}$. A similar procedure is applied for $\widetilde{\mathcal{G}}(t)=\mathfrak{c} \neq 1$; we find an exact expression for $t=T\left(r_{\mathrm{f}}\right)$, yet $r_{\mathrm{f}}$ is no longer computable in simple closed form (see Appendix D). By this computation, we find

$$
\begin{aligned}
r_{\mathrm{f}}(t) & \sim \sqrt{(4 \mathfrak{c}-1) t}, \\
h_{\mathrm{f}}(t) & \sim h_{\mathrm{f} 0}+r_{\mathrm{f} 0}-\frac{4 \mathfrak{c}}{\sqrt{4 \mathfrak{c}-1}} \sqrt{t} \quad \text { as } t \rightarrow \infty,
\end{aligned}
$$

where $\mathfrak{c}>1 / 2$. The height and slope profiles are computed from (5.11); evidently, $m(r, t)=\mathfrak{m}(\eta)$, where $\eta \sim$ const at the facet edge as $t \rightarrow \infty$. Note that

$$
m(r, t) \rightarrow m_{\mathrm{f}}=1-\frac{1}{4 \mathfrak{c}-1} \quad \text { as } r \downarrow r_{\mathrm{f}}(t), t \rightarrow \infty ;
$$

in particular, $m_{\mathrm{f}}=1 / 2$ if $\mathfrak{c}=3 / 4$ (see Remark 5.3 and section 7.1 ).

6. Numerical simulations. In this section, we provide numerical simulations to compare the discrete dynamics for M1 and M2 (without and with a geometry-induced step mobility) to continuum theories (with and without natural boundary conditions). The cases with $g=0$ and $g>0$ are presented separately since for $g=0$ we invoke exact continuum solutions, while for $g>0$, in the context of M2, we make use of numerically computed discrete data (collapse times $t_{n}$ ) in our modified boundary condition for the flux $\boldsymbol{\xi}$. Especially for $g>0$, we solve numerically PDE (4.9) for the slope profile by assuming self-similarity. In part of our numerics, we use as a starting point an approximate solution for the continuum slope that we extract heuristically via boundary layer theory if $0<g \ll 1$ [33]. We apply units with $\nu \Omega g_{1}=1=a$.

6.1. Numerics for $\boldsymbol{g}=\mathbf{0}$. Next, we solve (2.12) for $g=0$ under initial data (5.9) and compute the corresponding slopes $\mathcal{M}_{i}=1 /\left(r_{i+1}-r_{i}\right)$. In Appendix C, we derive exact solutions for the two top-step positions for validation of our numerics. We consider separately M1, where $\mathcal{G}_{i}(t) \equiv 1$ for an idealized step mobility (Appendix C.1), and M2 for a geometry-induced step mobility (Appendix C.2).

Copyright $@$ by SIAM. Unauthorized reproduction of this article is prohibited. 


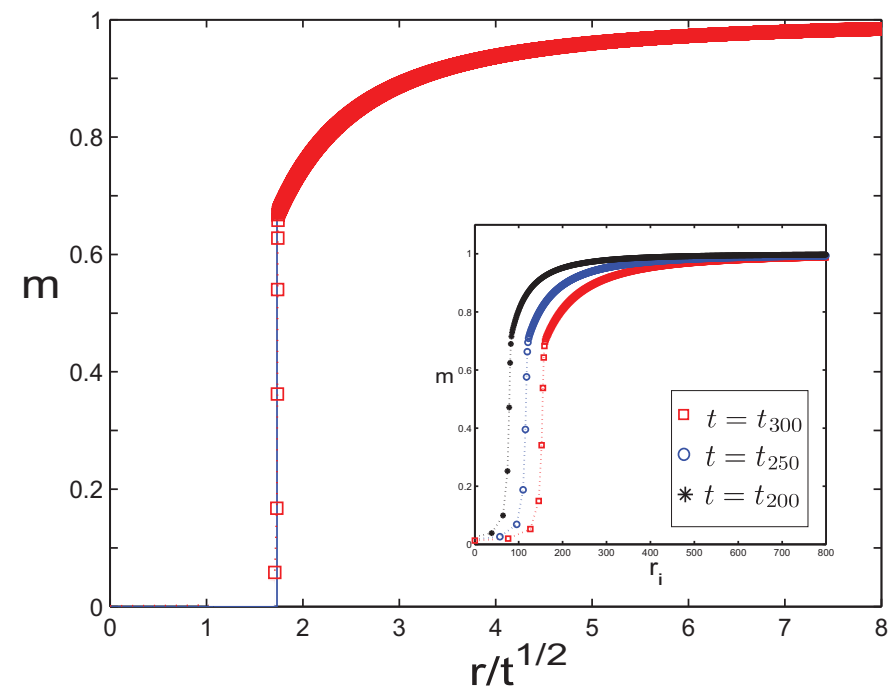

FIG. 6.1. Continuum slope $m(r, t)$ (solid line) and discrete slopes $\mathcal{M}_{i}(t)$ (symbols) as functions of $r / \sqrt{t}$ (where $r=r_{i}$ ) in the context of $M 1$ for $g=0$ and initial cone of unit slope with $N=9 \times 10^{3}$ steps. The slope $m(r, t)$ is computed from (5.12) with natural boundary conditions, and $\mathcal{M}_{i}(t)$ are obtained at $t=t_{n}$ by numerically solving $(2.12)$ with $\mathcal{G}_{i}(t) \equiv 1$. The vertical line indicates the facet position, $\eta_{\mathrm{f}}=r_{\mathrm{f}}(t) / \sqrt{t} \simeq \sqrt{3}$. Inset: Respective simulation data for $\mathcal{M}_{i}$ versus $r_{i}$ at collapse times $t=t_{n}(n=200,250,300)$, indicating self-similar behavior of $\mathcal{M}_{i}(t)$ for $n \gg 1$; the simulation data collapse to the graph of main figure.

6.1.1. Nongeometric step mobility (M1). In regard to the continuum slope, we invoke the natural boundary conditions (with a continuous flux $\boldsymbol{\xi}$ ). In Figure 6.1, we plot simulation data for $\mathcal{M}_{i}$ versus $r_{i}$ at different times $t=t_{n}$ and observe that the data collapse to a single graph if $r_{i}$ is scaled by $\sqrt{t}$. Figure 6.1 also shows that the numerically computed $\mathcal{M}_{i}(t)$ follow closely the predicted $m(r, t)$; in particular, the step simulations verify the predicted slope discontinuity at the facet edge where $m \rightarrow 2 / 3$ as $r \downarrow r_{\mathrm{f}}$ by $(5.15)$.

The self-similar behavior of the slope profile implies a scaling law for the step collapse times, $t_{n}$, for $n \gg 1$. We now give a heuristic argument (in the context of M1) for this scaling by combining the facet height drop due to step collapses with the natural boundary conditions. ${ }^{4}$ Requiring that the facet height decrease by multiples of the step size, $a=1$, we impose the relation $h_{\mathrm{f}}\left(t_{n-1}\right)-h_{\mathrm{f}}\left(t_{n}\right)=1[24,34]$, which we replace by $-\left(t_{n}-t_{n-1}\right) \dot{h}_{\mathrm{f}} \sim 1$ assuming $\left(t_{n}-t_{n-1}\right)\left|\ddot{h}_{\mathrm{f}}\right| \ll\left|\dot{h}_{\mathrm{f}}\right|$. By (5.3) and (5.5), we obtain that, for large $t, \delta t(t) \sim \frac{1}{2} r_{\mathrm{f}}(t)$, where $\delta t(t)=t_{n}-t_{n-1}$ is the continuum version of the step collapse time difference and $t_{n}=t \gg 1$. From (5.13) we obtain $\delta t(t) \sim \sqrt{3 t} / 2$, by which $t_{n} \sim(3 / 16) n^{2}$. This scaling law including the prefactor of $3 / 16$ is verified by our numerics; see Figure 6.2 , where the collapse times $t_{n}$ are plotted versus $n$. This observation provides additional evidence that for $g=0$, the natural boundary conditions are consistent with step flow in M1.

6.1.2. Geometry-induced step mobility (M2). Now set $g=0$ and $\mathcal{G}_{i}=$ $\left(r_{i}+r_{i-1}\right) /\left(2 r_{i}\right)$ in $(2.12)$ for the discrete dynamics. At the continuum scale, we invoke the slope from generalized boundary condition (5.6) with $\widetilde{\mathcal{G}}(t)=\mathfrak{c} \neq 1$. We consider two cases for $\mathfrak{c}$. First, we use the value of $\mathfrak{c}(\mathfrak{c} \simeq 0.766)$ extracted from

\footnotetext{
${ }^{4}$ For M2, a similar argument can be sketched but we do not pursue it here.
} 


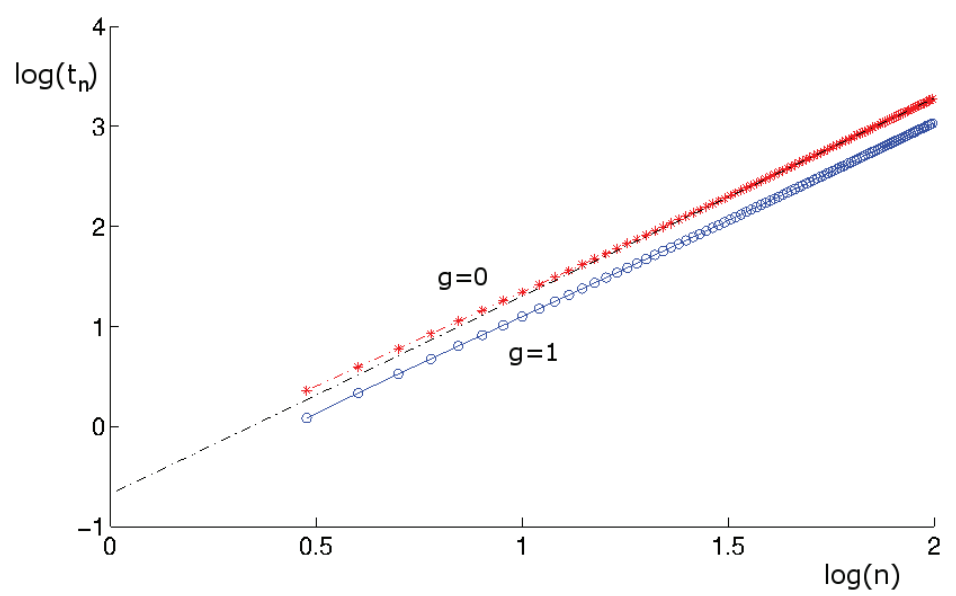

FIG. 6.2. Log-log plot of step collapse times $t_{n}$ versus $n$ for $g=0$ (asterisks) and $g=1$ (circles) in the context of $M 1$, numerically computed from $(2.12)$ for $\mathcal{G}_{i}(t) \equiv 1$ and initial cone of unit slope with $N=400$ steps. The dot-dashed (straight) line indicates the numerically computed large-n asymptotic behavior of $t_{n}$ for $g=0$. The scaling law $t_{n} \sim c n^{\beta}$ is verified for $n \gg 1$ with $\beta \approx 2$ for $g=0,1$. For $g=0$, we graphically find $c=0.1879 \approx 3 / 16$, in agreement with the analytical prediction via natural boundary conditions.

formula (5.7) combined with exact solutions for the two top-step ODEs, as shown in Appendix C.2. Alternatively, we use $\mathfrak{c}=3 / 4$, which we compute analytically by interpreting the facet height as shock and applying a Rankine-Hugoniot condition corresponding to a particular hyperbolic conservation law; see section 7.1.

Figures 6.3 and 6.4 show the comparison of continuum solutions to step simulations in the above two cases; the produced continuum slopes are practically indistinguishable, as expected. In particular, our step simulations predict that the slope at the facet edge approaches approximately the value $1 / 2$ as $r \downarrow r_{\mathrm{f}}$, consistent with (5.18) for $\mathfrak{c}=3 / 4$. Note that the continuum theory under natural boundary conditions fails to produce a slope consistent with step flow (see inset of Figure 6.3).

6.2. Numerics for $\boldsymbol{g}>\boldsymbol{0}$. In this subsection, we study PDE (4.9) via numerics for $g>0$ by assuming that the continuum slope is self-similar at long time. We are motivated by (i) the exact solution of section 5.4, where we found that $m(r, t)=\mathfrak{m}(\eta)$ with $\eta=r / \sqrt{t}$ and $r_{\mathrm{f}}(t)=\mathcal{O}(\sqrt{t})$ for $t \gg 1$; and (ii) our step simulations for $\mathcal{M}_{i}(t)$. Accordingly, we formally reduce the evolution PDE for $m(r, t)$ to an ODE for $\mathfrak{m}(\eta)$, solve this ODE numerically for $g>0$ under a boundary condition with a flux jump, and compare the continuum predictions to step simulations for $n \gg 1$.

First, we provide some numerical evidence that the discrete slopes have an apparently self-similar structure for large enough time. In Figure 6.5 (inset), we plot the discrete slopes, $\mathcal{M}_{i}(t)$, versus $r_{i}$ for $g=1$ at different collapse times, $t=t_{n}$, in the context of M1. We observe the data collapse once $r_{i}$ is scaled with $\sqrt{t}$, which indicates self-similarity of $\mathcal{M}_{i}(t)$. This observation is also made for other values of $g$ and for M2 via step simulations.

Next, we introduce similarity variables by writing $m(r, t) \sim t^{\omega} \mathfrak{m}(\eta)$ for $\eta=r t^{-\lambda}$ and large $t$; the real exponents $\lambda$ and $\omega$ are yet to be determined. By substitution into PDE (4.9) for $g>0$, elimination of $t$ from the coefficients yields $(\omega, \lambda)=(0,1 / 2)$, which is consistent with the discrete simulations. In particular, the value $\lambda=1 / 2$ implies the facet radius growth $r_{\mathrm{f}}(t)=\mathcal{O}(\sqrt{t})$; in this regime, the facet height should 


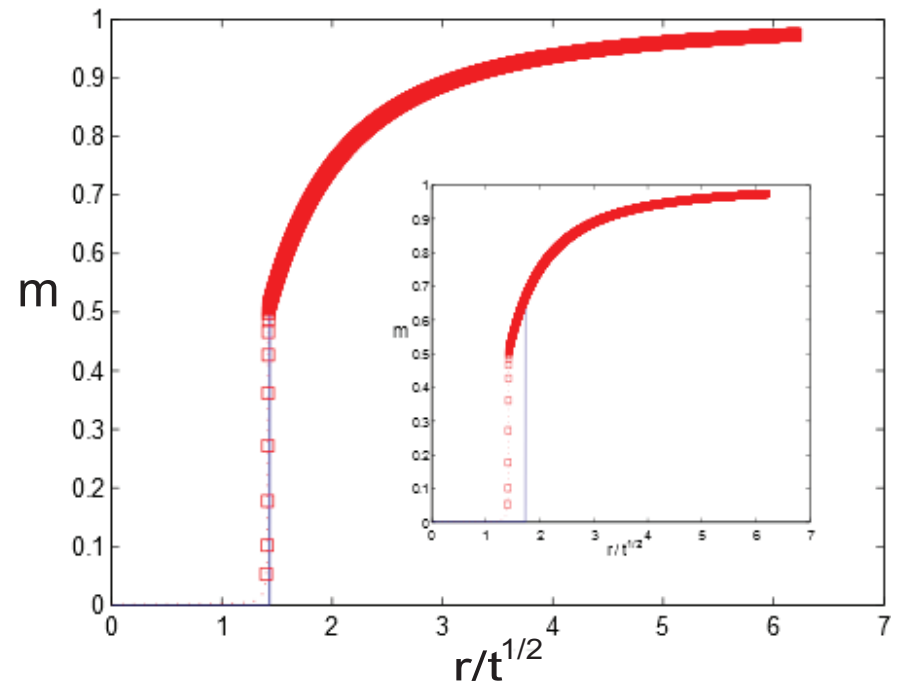

FIG. 6.3. Continuum slope $m(r, t)$ (solid line) and discrete slopes $\mathcal{M}_{i}(t)$ (symbols) as functions of $r / \sqrt{t}$ in the context of $M 2$ for long times $(n \gg 1), g=0$, and an initial cone of unit slope with $N=15 \times 10^{3}$ steps. The discrete slopes $\mathcal{M}_{i}(t)$ are determined from numerically solving at $t=t_{n}$ ODEs (2.12) with $\mathcal{G}_{i}=\left(r_{i}+r_{i-1}\right) /\left(2 r_{i}\right)$. In the main figure, the slope $m(r, t)$ is computed from (5.12) under jump condition (5.6) with (5.7) using an exact result for step collapse times (Appendix C.2). Inset: The same step simulation data for $\mathcal{M}_{i}$ in comparison to exact continuum slope (5.12) under natural boundary conditions.

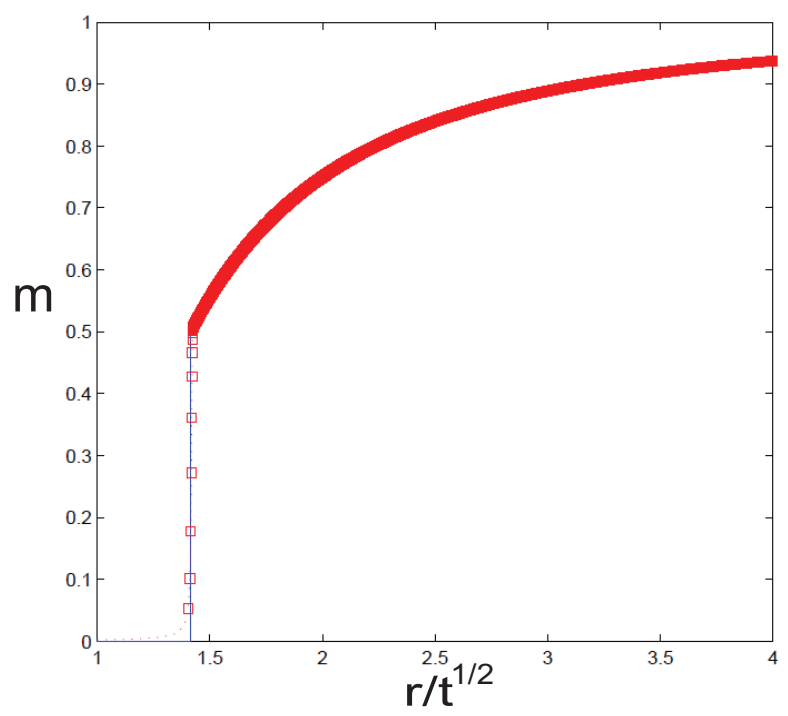

FiG. 6.4. Continuum slope $m(r, t)$ (solid line) and discrete slopes $\mathcal{M}_{i}(t)$ (symbols) as functions of $r / \sqrt{t}$ in the context of $M 2$ for long times $(n \gg 1), g=0$, and an initial cone of unit slope with $N=15 \times 10^{3}$ steps. The continuum slope $m(r, t)$ is now computed from (5.12) under jump condition (5.6) with $\widetilde{\mathcal{G}}(t)=3 / 4$ on the basis of a Rankine-Hugoniot condition for a hyperbolic conservation law (section 7.1). The discrete slopes $\mathcal{M}_{i}(t)$ are determined from numerically solving at $t=t_{n}$ ODEs (2.12) with $\mathcal{G}_{i}=\left(r_{i}+r_{i-1}\right) /\left(2 r_{i}\right)$, as in Figure 6.3 .

Copyright (c) by SIAM. Unauthorized reproduction of this article is prohibited. 


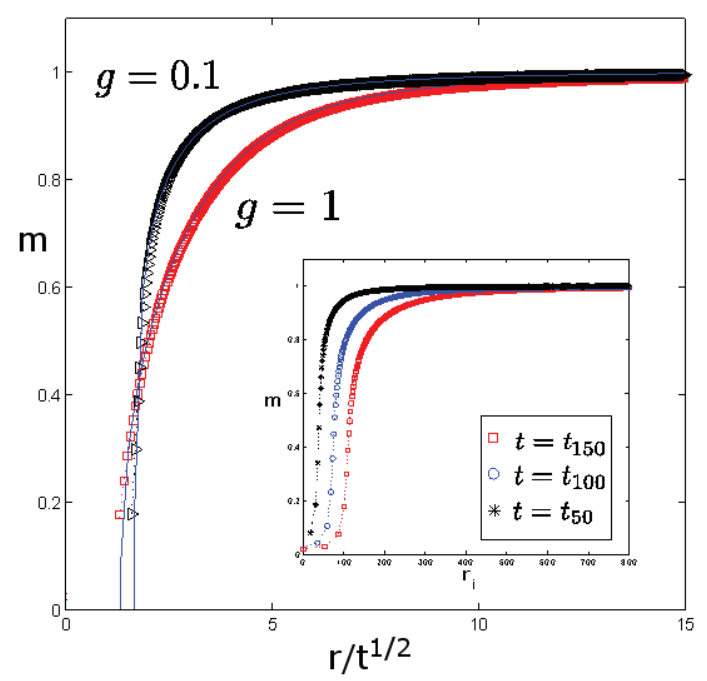

FIG. 6.5. Continuum slope $m(r, t)$ (solid line) and discrete slopes $\mathcal{M}_{i}(t)$ (symbols) for $M 1$ as functions of $r / \sqrt{t}$ for initial cone of unit slope with $N=9 \times 10^{3}$ steps; $g=0.1$ (triangles) and $g=1$ (squares). The slope $m(r, t)$ is computed from numerically solving (6.1)-(6.4) with $\mathfrak{c}=1(\breve{\mathfrak{c}}=1)$ stemming from natural boundary conditions, and $\mathcal{M}_{i}(t)$ are determined from $(2.12)$ with $\mathcal{G}_{i}(t)=1$. Inset: Discrete slopes $\mathcal{M}_{i}$ versus position $r=r_{i}$ at distinct collapse times $t=t_{n}(n=50,100,150)$ for $g=0.1$; the simulation data collapse to the graph of the main figure.

decay with $-\dot{h}_{\mathrm{f}}(t)=\mathcal{O}\left(t^{-1 / 2}\right)$. By condition (5.6), the factor $\widetilde{\mathcal{G}}(t)$ should become time independent; thus, we set $\widetilde{\mathcal{G}}(t)=\mathfrak{c}=$ const.

We proceed to describe the free-boundary problem for $\mathfrak{m}(\eta)$ in detail. PDE (4.9) away for the facet is converted to the ODE

$$
-\frac{1}{2} \eta \mathfrak{m}^{\prime}(\eta)=-\eta^{-2}+g\left[\eta^{-1}\left(\eta \mathfrak{m}^{2}\right)^{\prime}\right]^{\prime}, \quad \eta>\eta_{\mathrm{f}}:=r_{\mathrm{f}}(t) / \sqrt{t}
$$

here, $\eta_{\mathrm{f}}$ is unknown and the prime denotes differentiation with respect to $\eta$. Now we turn attention to boundary conditions for (6.1) by resorting to (5.2) and (5.4)-(5.6) with $\widetilde{\mathcal{G}}(t)=\mathfrak{c}$. By elimination of $\dot{h}_{\mathrm{f}}$, we find that $\mathfrak{m}(\eta)$ satisfies

$$
\begin{aligned}
& g\left(\eta \mathfrak{m}^{2}\right)^{\prime}=\breve{\mathfrak{c}}, \quad \eta \downarrow \eta_{\mathrm{f}}, \\
& \mathfrak{m}(\eta) \rightarrow 1, \quad \eta \rightarrow \infty, \\
& \mathfrak{m}(\eta)=0, \quad \eta \downarrow \eta_{\mathrm{f}} ; \quad \breve{\mathfrak{c}}:=2 \mathfrak{c}-1 .
\end{aligned}
$$

Note that there are three boundary conditions for a second-order ODE because $\eta_{\mathrm{f}}$ forms part of the solution. We have not been able to analytically integrate out (6.1)(6.4); hence, we proceed to find a solution numerically. We assume (but do not prove) that the self-similar slope is positive, $\mathfrak{m}(\eta)>0$ if $\eta>\eta_{\mathrm{f}}$.

To solve the (free-) boundary value problem of (6.1)-(6.4), we first apply a transformation of $(\eta, \mathfrak{m})$ that (i) maps $\left(\eta_{\mathrm{f}}, \infty\right)$ to a finite interval and (ii) renders linear the highest-order derivative in ODE $(6.1)[11,13]$. Simply apply a simple translation, $s=\eta-\eta_{\mathrm{f}}$. This maps $\left(\eta_{\mathrm{f}}, E\right)$ to $\left(0, \breve{E}=E-\eta_{\mathrm{f}}\right)$, where $E\left(E \gg \eta_{\mathrm{f}}\right)$ is a large number (which replaces infinity). In addition, we convert ODE (6.1) to a system of first-order 
ODEs for the variables $Y_{1}(s)=\mathfrak{m}^{2}(\eta)$ and $Y_{2}(s)=\left(\mathfrak{m}^{2}\right)^{\prime}(\eta), s \in(0, \breve{E})$,

$$
\begin{aligned}
& Y_{1}^{\prime}(s)=Y_{2}(s), \\
& Y_{2}^{\prime}(s)=\frac{1}{g}\left[\left(s+\eta_{\mathrm{f}}\right)^{-2}-\frac{1}{4}\left(s+\eta_{\mathrm{f}}\right) Y_{1}^{-1 / 2} Y_{2}\right]+\left(s+\eta_{\mathrm{f}}\right)^{-2} Y_{1}-\left(s+\eta_{\mathrm{f}}\right)^{-1} Y_{2},
\end{aligned}
$$

along with boundary conditions at $s=0$ (facet edge) and $s=\breve{E}$, obtained from (6.2)(6.4); in particular, $Y_{1}(\breve{E})=1$. It is of interest to seek the power series expansion ${ }^{5}$

$$
\mathfrak{m}(\eta)=Y_{1}(s)^{1 / 2} \sim \sum_{l=1}^{k} c_{l} s^{l / 2}=: S_{k}(s)
$$

which satisfies condition (6.4). By (6.2), we compute $c_{1}^{2}=\left(g \eta_{\mathrm{f}} / \breve{\mathfrak{c}}\right)^{-1}$. The remaining coefficients, $c_{l}=c_{l}\left(\eta_{\mathrm{f}}, g, \check{\mathfrak{c}}\right)$ for $l=2, \ldots, k$, are found via dominant balance in (6.1).

In our numerics for $\mathfrak{m}(\eta)$, we apply an iterative algorithm (e.g., the bvp4c MATLAB routine [23]) based on a suitable initial guess for $Y_{1}(s)$ that aims to satisfy $Y_{1}(\breve{E})=1[48]$. In this vein, we can make use of $(6.5)$ as we evaluate $Y_{1}(s)$ at a fixed point $s_{0}$ near 0 in terms of $\eta_{\mathrm{f}}$; then we choose $k=13$. A satisfactory approximate initial guess for $Y_{1}(s)$ for $0<g<1$, which apparently causes our numerical scheme to converge to a reasonable slope profile, is constructed through boundary layer theory $[22,33]$; see section 6.3 for related formulas.

6.2.1. Numerics for M1. In Figure 6.5, we plot the discrete slopes $\mathcal{M}_{i}$ for $M 1$ and the continuum slope $m$ under natural boundary conditions versus $r / \sqrt{t}$ for the values $g=0.1$ and 1 . Our numerical comparison shows that step flow is consistent with the predictions of the extended-gradient formalism.

In particular, we verify that the facet size decreases with $g=g_{3} / g_{1}$ at fixed time $t$. Physically, this effect can be attributed to the tendency of steps to cover a larger part of the surface if their repulsion $\left(g_{3}\right)$ increases or their self-energy (line tension, $g_{1}$ ) decreases; as a result, in principle any microscale event on top of the facet, i.e., collapse of a step, is expected to have a more pronounced effect on the macroscopic profile as $g$ becomes smaller (see, e.g., [34] for a model of DL kinetics).

We now derive a scaling law for the step collapse times, $t_{n}$, by combining the natural boundary conditions with the discrete facet height drop (see section 6.1.1). By $\delta t(t) \sim \frac{1}{2} r_{\mathrm{f}}(t)$, where $\delta t(t)=t_{n}-t_{n-1}$ and $t_{n}=t \gg 1$, we find $t_{n}-t_{n-1} \sim C(g) \sqrt{t}$; thus, $t_{n} \sim C^{2} n^{2} / 4$ for $n \gg 1$ (cf. Figure 6.2 for $g=1$ ). This $C(g)$ should decrease with $g$ since stronger step repulsions cause steps to shrink faster on top of the facet [34]; this monotone behavior is verified by our numerics.

6.2.2. Numerics for M2. In Figures 6.6 and 6.7, we compare step simulations for M2 to predictions of continuum theories for distinct values of $g(g=0.1$ and 1 ). Evidently, the generated discrete slopes are in agreement with the macroscopic slope coming from a flux jump, condition (5.6) with (5.7), where the requisite $t_{n}$ are computed from simulations. By contrast, the continuum slope stemming from natural boundary conditions (including flux continuity) deviates from step simulation data (see inset of Figure 6.6).

\footnotetext{
${ }^{5}$ Expansion (6.5) is only postulated. This expansion is consistent with the structure of the evolution PDE. A similar expansion is derived formally in [36] by direct reduction of discrete schemes to an integral equation for $m$ and subsequent iteration for the setting of evaporation dynamics of a 1D step train connecting two semi-infinite facets. We do not pursue a rigorous justification for (6.5).
} 


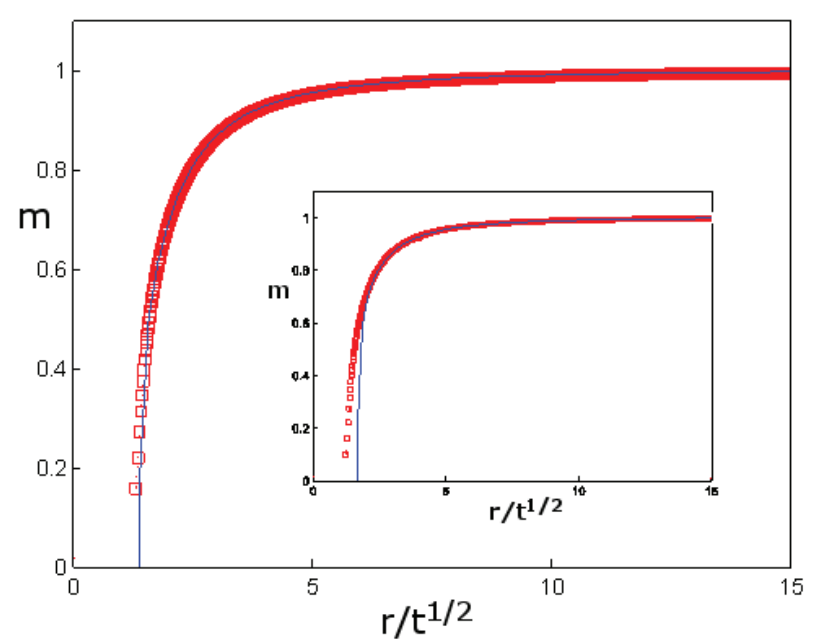

FIG. 6.6. Continuum slope $m(r, t)$ (solid line) and discrete slopes $\mathcal{M}_{i}$ (symbols) for $M 2$ as functions of $r / \sqrt{t}$ for long times, $g=0.1$, and initial cone of unit slope with $N=7 \times 10^{3}$ steps. The discrete slopes $\mathcal{M}_{i}(t)$ are determined from numerically solving ODEs $(2.12)$ with $\mathcal{G}_{i}=\left(r_{i}+\right.$ $\left.r_{i-1}\right) /\left(2 r_{i}\right)$. Main figure: The continuum-scale slope, $m(r, t)$, is computed from numerically solving (6.1)-(6.4) with $\breve{\mathfrak{c}}$ from (5.7), accounting for flux jump. Inset: With the same step simulation data, the continuum-scale slope $m(r, t)$ is computed from flux continuity for $\breve{\mathfrak{c}}=1$.

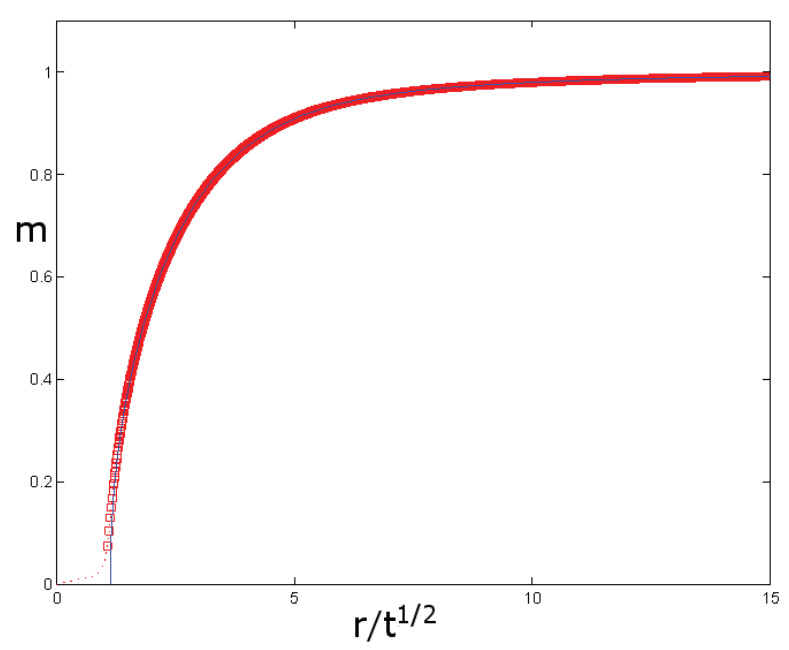

FIG. 6.7. Continuum slope $m(r, t)$ (solid line) and discrete slopes $\mathcal{M}_{i}$ (symbols) for $M 2$ as functions of $r / \sqrt{t}$ for long times, $g=1$, and initial cone of unit slope with $N=10^{4}$ steps. The discrete slopes $\mathcal{M}_{i}(t)$ are determined from ODEs (2.12) with $\mathcal{G}_{i}=\left(r_{i}+r_{i-1}\right) /\left(2 r_{i}\right)$. The continuumscale slope, $m(r, t)$, is computed from numerically solving (6.1)-(6.4) with $\tilde{\mathfrak{c}}$ from (5.7), accounting for a flux jump at the facet edge.

6.3. Approximation by boundary layer theory. In this subsection, we formally construct an approximate solution to the free-boundary problem described by PDE (4.9) with conditions (5.2) and (5.4)-(5.6) if $0<g \ll 1$. The assumption of self-similarity is not necessary in principle. The main observation is that by setting $g=0$ in the evolution PDE (4.9) it is impossible to obey the continuity of slope, (5.5). So, we split the domain outside the facet into two regions: an "outer" region, in 
which the step self-energy (line tension) dominates over the step interaction energy, and the "inner" region (boundary layer), in which the step interaction energy is significant [33]. The width of the boundary layer should scale with a positive power of $g$. Inside the boundary layer, the slope $m$ varies smoothly from its zero value $(m=0)$ at the facet edge to the behavior predicted for $g=0$ in the outer region; cf. (5.12). In general, the outer solution should be compatible with the continuity of height, flux jump, and far field condition. Hence, we only need to compute the inner solution by imposing zero slope at the facet edge. Here, we provide only the final formulas; details of our boundary layer analysis are given in Appendix E.

We start by defining the inner variable

$$
\zeta=\frac{r-r_{\mathrm{f}}(t)}{g^{\alpha} w(t)}
$$

where $g^{\alpha} w(t)$ measures the (a priori unknown) width of the boundary layer, $\alpha$ is a positive exponent (to be determined), and $r_{\mathrm{f}}(t)=r_{\mathrm{f}}(t ; g=0)$ denotes the facet radius for $g=0 ; \zeta=\mathcal{O}(1)$ inside the layer. After some algebra, we find (see Appendix E)

$$
\alpha=1, \quad w(t)=\dot{r}_{\mathrm{f}}(t)^{-1}\left[1-\frac{t}{r_{\mathrm{f}}(t)^{2}}\right],
$$

where formulas for $r_{\mathrm{f}}(t)$ are provided in section 5.4 and Appendix D. A composite formula for the slope away from the facet reads

$$
m(r, t) \sim 1-\frac{t}{r^{2}}+a_{0}(t)\left[f_{0}(\zeta)-1\right] \quad r>r_{\mathrm{f}}(t), \quad a_{0}(t)=1-\frac{t}{r_{\mathrm{f}}(t)^{2}},
$$

which encompasses both outer and inner solutions. The function $a_{0}(t)$ is the limiting value of the outer slope at the facet edge, while $f_{0}(\zeta)$ for the inner solution satisfies

$$
f_{0}(\zeta)=1-e^{-\zeta / 2} e^{-f_{0}(\zeta)}, \quad \zeta>0 .
$$

Note that $1-f_{0}(\zeta)$ decays exponentially as $\zeta \rightarrow \infty$; thus, (6.7) properly reduces to the continuum solution for $g=0$ outside the boundary layer. Formulas (6.6) and (6.7) can be simplified for $t \gg 1$ by use of asymptotic formula (5.13), under flux continuity, or, more generally, formula (5.16), which includes a flux jump.

7. Discussion. In this section, we discuss the interpretation of the facet height as a type of shock. This perspective is promising for showing convergence of discrete schemes with collapsing layers but remains largely unexplored. Our starting point is to view the continuum step position, $R$, as a function of height and time. In section 7.1, we apply speculation and heuristics: We point out for $g \geq 0$ that the flux $(\boldsymbol{\xi})$ continuity, which is consistent with step flow in M1, can be read as a certain Rankine-Hugoniot-type condition. Our discussion becomes more concrete for noninteracting steps $(g=0)$ : We present a family of hyperbolic conservation laws and identify the shock accordingly. In section 7.2 , we prove that for $g=0$ the solutions to M1 and M2 converge to entropy solutions of conservation laws from the above family.

7.1. Facet height as shock. Consider PDE (4.7) away from the facet. Using Lagrangian variables $[12,13]$, we view the step radius, $R$, as a function of height, $h$, and $t$. Let $\chi=h_{\mathrm{f}}(0)-h$, which measures the height relative to the initial surface structure in the (physical) domain with $h<h_{\mathrm{f}}(t)$, and set $R=R(\chi, t)$ in this domain. 
By the level set motion law $\partial_{t} R=\partial_{t} h / m$, where $m=\left(\partial_{\chi} R\right)^{-1}$, PDE (4.7) reads

$$
\partial_{t}\left(R^{2}\right)+2 \Omega \nu g_{1} \partial_{\chi}\left[R\left(1+g\left(\partial_{\chi} R\right)^{-2}\right)\right]=0, \quad \chi>\chi_{\mathrm{f}}(t):=h_{\mathrm{f}}(0)-h_{\mathrm{f}}(t)
$$

assuming monotone increasing $R(\cdot, t)$. It should be stressed that this PDE holds and is consistent with step flow away from the facet, and in principle it breaks down as $\chi$ approaches $\chi_{\mathrm{f}}$ from above because of discrete effects.

Suppose a continuum theory that is consistent with steps is formulated everywhere. Intuitively, in our models such a theory would have to be compatible with an extension of the domain of $R(\cdot, t)$ via $R(\chi, t) \equiv 0$ for $\chi<\chi_{\mathrm{f}} ; R(\cdot, t)$ would acquire a jump at $\chi=\chi_{\mathrm{f}}$. We expect this property to emerge from the discrete schemes since the initial data and solutions of ODEs for M1 and M2, where step radii are set to zero after their collapse times, appear consistent with this prescription. A difficulty is that this jump in principle cannot be derived from existing fully continuum principles. We view this speculated discontinuity, and any related jump of functions of $R$, as a shock-like wave in $(\chi, t)$; the shock speed is $\dot{\chi}_{\mathrm{f}}(t)=-\dot{h}_{\mathrm{f}}(t)$, the speed of the facet, which decays with time.

It is now tempting to claim that the boundary condition for the flux $\boldsymbol{\xi}(r, t)$ at the facet edge corresponds to a Rankine-Hugoniot-type condition for the shock speed. Specifically, for M1 we write PDE (7.1) as the conservation statement $\partial_{t} \varrho+\partial_{\chi} \mathfrak{J}=$ 0 with "density" $\varrho=\pi R^{2}$ (step area) and associated "flux" $\mathfrak{J}=(2 \pi R) \nu \Omega g_{1}[1+$ $\left.g\left(\partial_{\chi} R\right)^{-2}\right]$ for $\chi>\chi_{\mathrm{f}}$ and define $\varrho=\pi R^{2} \equiv 0$ and $\mathfrak{J} \equiv 0$ for $\chi<\chi_{\mathrm{f}}$. Then $\boldsymbol{\xi}$-continuity (5.3) is equivalent to $\dot{\chi}_{\mathrm{f}}=[\mathfrak{J}] /[\varrho]$ at $\chi=\chi_{\mathrm{f}}(t)$, where $[\varrho]$ denotes the jump of $\varrho$; the strength of the shock is proportional to the facet area, which grows with time. ${ }^{6}$

These considerations become more concrete for $g=0$, as shown in section 7.2. In this case, for smooth $R(\chi, t)$ we can generate a family of hyperbolic conservation laws via multiplication of (7.1) by $R^{\varsigma-1}$ with $\varsigma>0$ :

$$
\partial_{t}\left(R^{\varsigma+1}\right)+\Omega \nu g_{1}\left(\frac{\varsigma+1}{\varsigma}\right) \partial_{\chi}\left(R^{\varsigma}\right)=0, \quad \chi>\chi_{\mathrm{f}}(t) .
$$

The definition of the facet height as shock follows naturally: Extend $R(\chi, t)$ to all real $\chi$ by replacing (7.2) by its weak formulation, assuming initial data with $R(\chi, 0) \equiv 0$ for $\chi<\chi_{\mathrm{f}}(0)=0$. In other words, we artificially extend the evolution PDE to the vapor $\left(h>h_{\mathrm{f}}\right)$ by stating that no steps exist there. It turns out that, among all possible weak solutions of (7.2) compatible with the given initial data [29], step flow is consistent with a shock wave with $R \equiv 0$ for $\chi<\chi_{\mathrm{f}}(t)$; in particular, the continuum limit of M1 (M2) corresponds to (7.2) with $\varsigma=1(\varsigma=2)$. The shock-wave strength is $r_{\mathrm{f}}(t)^{\varsigma+1}$. The precise statement regarding convergence is deferred to section 7.2.

In particular, we write the Rankine-Hugoniot condition as

$$
-\dot{h}_{\mathrm{f}}(t)=\Omega \nu g_{1}\left(\frac{\varsigma+1}{\varsigma}\right) r_{\mathrm{f}}(t)^{-1}
$$

The corresponding weak solution, $u(\chi, t)=R(\chi, t)^{\varsigma+1}$, satisfies an entropy condition; thus, it is the unique entropy solution to (7.2) [29]. In particular, for $\varsigma=1,(7.3)$ is precisely the continuity of $\boldsymbol{\xi}$ in the subgradient formulation with $g=0$. Furthermore,

\footnotetext{
${ }^{6}$ The jump of $R(\cdot, t)$, which is present in M1 and M2, should not be confused with the jump of $\boldsymbol{\xi}$, which is introduced for M2 and vanishes in M1. The shock-like wave refers to the former jump.
} 
for $\varsigma=2,(7.3)$ produces a discontinuous flux $\boldsymbol{\xi}$ at the facet edge; a comparison to condition (5.6) entails $\widetilde{\mathcal{G}}(t)=3 / 4$. Our simulations (Figures 6.1 and 6.4 ) indicate that step flow is consistent with the above shock-wave interpretation.

7.2. Convergence of discrete schemes for $\boldsymbol{g}=\mathbf{0}$. Next, we show that for $g=0$ and linear initial data for the step radii, $r_{i}$, the solution to each of M1 and M2 is the entropy solution of (7.2) with $\varsigma=1$ and 2 , respectively. In the remainder of this section, we adopt the standard formalism of difference schemes for conservation laws [30,31], assuming that the reader has some familiarity with numerical methods. We apply units with $\Omega \nu g_{1}=1$; the step height $a$ is the mesh size (with $a \rightarrow 0$ ). In this section and Appendix F, the surface structure is assumed to be finite (i.e., it is contained in a bounded region) so that a compactness theorem can be invoked. In particular, we restrict the domain of $(7.3)$ to $\chi \leq h_{f}(0)$. We note in passing that solving (7.3) does not require any condition at this boundary since we use characteristics.

Consider ODEs (2.12) with $g=0$ (and replace $i$ by $j$ ). We extend the discrete dynamics to all integer $j$ by extending the initial data for $r_{j}$ to 0 for $j<0$. The ODEs for both M1 and M2 are recast to the conservative form

$$
\dot{U}_{j}=-\frac{1}{a}[F(U(t) ; j)-F(U(t) ; j-1)],
$$

where $U_{j}(t)=U^{a}\left(\chi_{j}, t\right)$ is the discrete solution variable, which equals $r_{j}^{2}$ for M1 and $r_{j}^{3}$ for $\mathrm{M} 2$, with $\chi_{j}=j a$ (mesh in $\left.\mathbb{R}\right), \chi_{N}=N a=h_{f}(0)$, and $U=U^{a}$ denotes the $a$-dependent, infinite-dimensional vector with components $U_{j}$. (The fact that the finite surface structure ends at $\chi=h_{f}(0)$ does not alter the solution of (7.4) because the ODE for $U_{j}$ depends only on $U_{i}$ for $i \leq j$.) The numerical flux $F$ is defined by

$$
F(U(t) ; j)=\left\{\begin{array}{lr}
c U_{j}(t)^{\mathfrak{b}}, & U_{j}>0, \\
0, & U_{j} \leq 0,
\end{array}\right.
$$

where $0<\mathfrak{b} \leq 1$ and $c$ is a positive constant; $(\mathfrak{b}, c)=(1 / 2,2)$ for M1 and $(\mathfrak{b}, c)=$ $(2 / 3,3 / 2)$ for M2. In the following analysis, set $c=1$ without loss of generality. $F$ is Hölder-continuous with exponent $\mathfrak{b}$. It is also useful to define $\chi_{j \pm 1 / 2}=(j \pm 1 / 2) a$.

One of our goals is to show that the solution to (7.4) with (7.5) and $\mathfrak{b}=\varsigma /(\varsigma+$ 1) converges to a weak solution of conservation law (7.2). Specifically, we aim to prove that, as $a \rightarrow 0, U^{a}(t)$ converge (weakly) to $u(\chi, t)=R(\chi, t)^{\varsigma+1}$ that is the entropy solution to (7.2). Equation (7.4) with (7.5) is amenable to known methods for the convergence of conservative difference schemes [31]. The numerical flux, $F$, is "consistent" with the respective conservation law if we extend the definition of "consistency" in [31] to include Hölder-continuous functions with exponents in $(0,1]$.

We can show that for M1 and M2 there exists a convergent sequence in $\left\{U^{a}\right\}$ as $a \rightarrow 0$ (see Lemma F.1 in Appendix F). Based on this property, our core result is the following.

Proposition 7.1. Let $U(t)=U^{a}(t)$ be a solution of numerical scheme (7.4) with flux (7.5) and initial data that is compactly supported and monotone increasing within its support. Then, $U^{a}(t)$ converges to the unique entropy solution $u(\chi, t)$ of the conservation law $\partial_{t} u+\partial_{\chi} f(u)=0$ with flux

$$
f(u)= \begin{cases}u^{\mathfrak{b}}(t), & u>0, \\ 0, & u \leq 0,\end{cases}
$$

and initial data $u(\chi, 0)$ compatible with the discrete initial data. 
The proof of Proposition 7.1 is technical but uses standard techniques [31]; it is therefore deferred to Appendix F.

In the statement of Proposition 7.1, the term "compatible" is used to mean that the initial discrete data stems from the cell average of the continuum-scale data [31]. The linear initial data for $r_{j}(t)$ and $h(r, t)$ of section 5.3 satisfy this property: By setting $u(\chi, t)=R(\chi, t)^{\varsigma+1}$ (by the notation of section 7.1) and $\beta=\varsigma+1$, we have

$$
\int_{\chi_{j-1 / 2}}^{\chi_{j+1 / 2}} u(\chi, 0) \mathrm{d} \chi=\int_{\chi_{j-1 / 2}}^{\chi_{j+1 / 2}} \chi^{\beta} \mathrm{d} \chi=\left.\frac{\chi^{\beta+1}}{\beta+1}\right|_{\chi_{j}-\frac{1}{2} a} ^{\chi_{j}+\frac{1}{2} a}=a\left[\chi_{j}^{\beta}+\mathcal{O}(a)\right] .
$$

Note that the proof of convergence followed here does not seem applicable to the case with nonzero step interactions $(g>0)$. Difficulties include the definition of numerical flux, apparent unboundedness of the step chemical potential, and the effect of finite $N$. The case with nonzero $g$ is the subject of work in progress.

8. Conclusion. We studied the continuum limits of two discrete schemes, M1 and M2, for a train of descending steps with a facet in the radial setting. In these schemes, the step velocity is driven by the step chemical potential under evaporationcondensation kinetics. Specifically, in M1 the discrete mobility is simply proportional to the upper terrace width. In M2, this mobility acquires a multiplicative, geometryinduced, factor consistent with a limit of the BCF model; this factor accentuates the influence of extremal steps. Away from the facet, the step ODEs reduce to the same second-order, quasi-linear parabolic PDE by which the height flow is the first variation of a singular surface free energy. By assuming initial data for a linear cone, we focused on the numerical comparison at long time of discrete slopes to the continuum-scale slope under different sets of boundary conditions at the facet edge.

Our main achievement was to show how geometry and kinetics of extremal steps play a crucial role in the formulation of continuum limits (PDE and boundary conditions at the facet) consistent with step flow. At the continuum scale, our starting point was the well-known subgradient theory by which a unique "highly regular" solution is sought via continuity of height, slope, and a flux expressing mass conservation. By assuming self-similarity of continuum slopes, we showed that the subgradient formalism is compatible with M1 but not M2. Our numerical computations suggest that for consistency of the continuum theory with M2, one of the natural boundary conditions should be modified; the flux acquires a discontinuity at the facet boundary. This jump was expressed in terms of values of the geometry-induced factor for extremal steps at step collapse times. Our work suggests a precise mechanism through which extremal steps can influence macroscopic profiles; the collapses of such steps can cause a discrepancy between discrete and thermodynamics-based continuum predictions.

To the best of our knowledge, this is the first time discrete effects on top of facets are incorporated into existing boundary conditions of the continuum thermodynamics approach. We believe that this treatment opens up new possibilities; for example, it is promising for non-self-similar settings and appears less sensitive to errors in the computation of collapse times than the previous method of injecting discreteness directly into the facet speed $[24,34]$. However, our treatment is so far applied to two step models of evaporation kinetics in the self-similarity regime. The relevance of this approach to other step models, especially in surface diffusion where the evolution PDE is of fourth order, and full time-dependent settings should be explored.

In light of our numerical results, we discussed how the facet may be linked to a shock-like wave in Lagrangian coordinates; the shock strength and speed are directly 
related to the facet radius and speed, respectively. The idea of viewing facets as shocks is not new; in $[13,14,15,16,52] \mathrm{PDE}$ solutions are connected to shocks in the continuum scale. Here, we aim to connect shock-like solutions to step dynamics. In section 7 , we placed the shock notion on a firm basis for noninteracting steps, proving that the discrete solution to each of M1 and M2 converges to the (unique) entropy solution of a respective conservation law. Our discussion suggests that a methodology for continuum limits of step flow, when steps collapse on top of facets, could rely on generalized (nonsmooth) solutions of evolution PDEs (e.g., conservation laws). In this approach, the continuum step position, $R$, as a function of height satisfies a certain $\mathrm{PDE}$ on one side of a discontinuity (away from the facet) and may vanish on the other side (where the PDE may not hold); a difficulty for interacting steps is to connect the two values of $R$ via a condition which may include discrete effects. This perspective allows for boundary conditions broader than the natural ones. However, key questions are left unresolved, e.g., the convergence of our discrete schemes for interacting steps.

Some other open problems deserve attention. The nature of self-similar discrete and continuum solutions in our radial setting needs to be addressed rigorously. Our study, so far limited to radial geometries with conical initial data, should be extended to a richer class of initial data, other step kinetics (e.g., the DL and ADL regimes), and the full two-dimensional geometry where steps may no longer be circles. A difficulty in two-dimensional geometries is the possibility of meandering instabilities; to the best of our knowledge, there is no widely accepted measure of meandering for closed steps, say, perturbations of circles.

Appendix A. Discrete mobility from limit of BCF-type model. In this appendix, we discuss a plausible origin of the geometric factor $\mathcal{G}_{i}$ in M2 by recourse to a special limit of surface diffusion with desorption and negative ES barrier $[44,47]$ in the radial geometry. The main guiding principle is offered by the BCF model [5].

Let $C_{i}$ be the concentration of adatoms on the $i$ th terrace, $r_{i-1}<r<r_{i}$, and $t_{\mathrm{ds}}$ be a typical desorption time. In juxtaposition to our ad hoc models M1 and M2, here we adopt the viewpoint that the step velocity is driven by changes in the adatom flux across terraces. So, we start with a diffusion equation for the concentration, $C_{i}$, of adatoms including desorption under the quasi-steady approximation:

$$
\partial_{r r} C_{i}+r^{-1} \partial_{r} C_{i}-\kappa^{2} C_{i}=0, \quad r_{i-1}<r<r_{i},
$$

where $\kappa^{2}=\left(D_{s} t_{\mathrm{ds}}\right)^{-1}(\kappa>0)$. Equation (A.1) has the general solution [53]

$$
C_{i}(r)=A_{i} I_{0}(\kappa r)+B_{i} K_{0}(\kappa r),
$$

where $I_{0}(z)$ and $K_{0}(z)$ are modified Bessel fuctions of zeroth order and $A_{i}$ and $B_{i}$ are integration constants to be determined from the boundary conditions at the bounding step edges. The requisite conditions are

$$
\begin{aligned}
-\mathcal{J}_{i}(r, t) & =k_{u}\left(C_{i}-C_{i}^{\mathrm{eq}}\right), & & r=r_{i}, \\
\mathcal{J}_{i}(r, t) & =k_{d}\left(C_{i}-C_{i+1}^{\mathrm{eq}}\right), & & r=r_{i+1},
\end{aligned}
$$

where $\mathcal{J}_{i}(r, t)=-D_{s} \partial_{r} C_{i}(r, t)$ is the adatom flux on the $i$ th terrace, $k_{u}\left(k_{d}\right)$ is the kinetic rate for atom attachment/detachment at an up-step (down-step), and $C_{i}^{\text {eq }}$ is 
the equilibrium concentration at the $i$ th step edge. By (A.2) and (A.3), we obtain

$$
\begin{aligned}
A_{i}=\frac{1}{\mathcal{D}_{i}} & \left\{-\frac{k_{u}}{D_{s} \kappa} C_{i}^{\mathrm{eq}}\left[K_{0}^{\prime}\left(\kappa r_{i+1}\right)+\frac{k_{d}}{D_{s} \kappa} K_{0}\left(\kappa r_{i+1}\right)\right]\right. \\
& \left.-\frac{k_{d}}{D_{s} \kappa} C_{i+1}^{\mathrm{eq}}\left[K_{0}^{\prime}\left(\kappa r_{i}\right)-\frac{k_{u}}{D_{s} \kappa} K_{0}\left(\kappa r_{i}\right)\right]\right\}, \\
B_{i}=\frac{1}{\mathcal{D}_{i}} & \left\{\frac{k_{d}}{D_{s} \kappa} C_{i+1}^{\mathrm{eq}}\left[I_{0}^{\prime}\left(\kappa r_{i}\right)-\frac{k_{u}}{D_{s} \kappa} I_{0}\left(\kappa r_{i}\right)\right]\right. \\
& \left.+\frac{k_{u}}{D_{s} \kappa} C_{i}^{\mathrm{eq}}\left[I_{0}^{\prime}\left(\kappa r_{i+1}\right)+\frac{k_{d}}{D_{s} \kappa} I_{0}\left(\kappa r_{i+1}\right)\right]\right\},
\end{aligned}
$$

where the prime denotes differentiation with respect to the argument and

$$
\begin{aligned}
\mathcal{D}_{i}= & {\left[I_{0}^{\prime}\left(\kappa r_{i}\right)-\frac{k_{u}}{D_{s} \kappa} I_{0}\left(\kappa r_{i}\right)\right]\left[K_{0}^{\prime}\left(\kappa r_{i+1}\right)+\frac{k_{d}}{D_{s} \kappa} K_{0}\left(\kappa r_{i+1}\right)\right] } \\
& -\left[I_{0}^{\prime}\left(\kappa r_{i+1}\right)+\frac{k_{d}}{D_{s} \kappa} I_{0}\left(\kappa r_{i+1}\right)\right]\left[K_{0}^{\prime}\left(\kappa r_{i}\right)-\frac{k_{u}}{D_{s} \kappa} K_{0}\left(\kappa r_{i}\right)\right] .
\end{aligned}
$$

The step velocity law for surface diffusion reads $[5,26]$

$$
\dot{r}_{i}=\frac{\Omega}{a}\left(\mathcal{J}_{i-1}-\mathcal{J}_{i}\right), \quad r=r_{i} .
$$

By (A.2), the step velocity becomes

$$
\dot{r}_{i}=-\frac{\Omega}{a} \kappa D_{s}\left[A_{i-1} I_{0}^{\prime}\left(\kappa r_{i}\right)+B_{i-1} K_{0}^{\prime}\left(\kappa r_{i}\right)-A_{i} I_{0}^{\prime}\left(\kappa r_{i}\right)-B_{i} K_{0}^{\prime}\left(\kappa r_{i}\right)\right] .
$$

We now simplify the right-hand side of (A.6) under the conditions

$$
k_{u} t_{\mathrm{ds}} \ll r_{i}, \quad \kappa r_{i} \ll 1, \quad k_{u} \ll k_{d}, \quad\left|r_{i}-r_{i-1}\right| \ll k_{d} t_{\mathrm{ds}} .
$$

Note that the second inequality implies that the diffusion length $\sqrt{D_{s} t_{\mathrm{ds}}}$ is large compared to the step radius. The third inequality expresses an inverse ES effect. Thus, we obtain the simplified step motion law

$$
\dot{r}_{i}=-\frac{\Omega}{t_{\mathrm{ds}}} \frac{r_{i}+r_{i-1}}{2 r_{i}} \frac{r_{i}-r_{i-1}}{a} C_{i}^{\mathrm{eq}}
$$

which reveals the (geometric) structure of the discrete mobility if $C_{i}^{\mathrm{eq}}$ is affine in the step chemical potential, $\mu_{i}$. Now recall the usual Gibbs-Thomson relation [26], $C_{i}^{\text {eq }}=C_{\mathrm{s}} \exp \left(\mu_{i} / \mathcal{T}\right) \sim\left(C_{\mathrm{s}} / \mathcal{T}\right)\left(\mathcal{T}+\mu_{i}\right)$ for $\left|\mu_{i}\right| \ll \mathcal{T}$, where $\mathcal{T}$ is Boltzmann's energy (or absolute temperature in appropriate units); evidently, M2 results from (A.8) by removal of the constant $\mathcal{T}$ [cf. (2.4)].

Appendix B. Elements of subgradient formalism. In this appendix, we briefly describe elements of the subgradient theory, assuming the reader has some familiarity with basic functional analysis. The subgradient formalism provides a means of analyzing evolution laws that have a steepest descent structure with respect to a convex, singular energy functional [27]. An elementary exposition for the surface diffusion case can be found in [42].

Formally speaking, the notion of the subgradient extends the concept of conventional gradient (or derivative) to convex functions or functionals that are not necessarily differentiable everywhere. Let $\mathcal{H}$ be a Hilbert space and $F$ be a convex functional 
on $\mathcal{H}$. The subdifferential, $\partial F(x)$, of $F$ at the point $x$ of $\mathcal{H}$ is the set of all vectors $v$ in $\mathcal{H}$ that satisfy the inequality

$$
F(x+h)-F(x) \geq\langle v, h\rangle \quad \text { for all } h \text { in } \mathcal{H},
$$

where $\langle v, h\rangle$ denotes the inner product of $\mathcal{H}$. We call such $v$ the subgradient of $F(x)$.

Consider first the classic example of the convex function $f(x)=|x|$, where $-1 \leq$ $x \leq 1$. In this case, $\mathcal{H}$ is the $1 \mathrm{D}$ space $[-1,1]$ equipped (trivially) with the product of reals. Since $f(x)$ is differentiable at $x \neq 0$, we find $\partial f(x)=\{\operatorname{sgn}(x)\}$, a singleton, where $\operatorname{sgn}(x)=x /|x|$ is the sign function. The notion of $\partial f(x)$ becomes particularly useful for $x=0$, where $f(x)$ is not differentiable. To compute $\partial f(0)$, one notices that for any real $h, f(h)-f(0)=|h| \geq|\varpi h|$ only if $|\varpi| \leq 1$. It is easily deduced that $\partial f(0)=[-1,1]$, the set of all possible slopes of linear graphs bounded above by the graph of $y=|x|$ in the $x y$ plane. This example can be extended to $d$ space dimensions: Consider $f(\mathbf{x})=|\mathbf{x}|$, where $\mathbf{x}$ is any point in the $d$-dimensional Euclidean space, $\mathbb{R}^{d}$; then, $\partial f(\mathbf{x})=\{\mathbf{x} /|\mathbf{x}|\}$ if $\mathbf{x} \neq 0$, and $\partial f(0)=B^{d}(0,1)$.

The above ideas can be generalized to functionals, i.e., mappings of vectors in $\mathcal{H}$ to real numbers, or more generally to its underlying algebraic field. An abstract formulation suggests that an evolution with the variational structure can be viewed as "trajectories" of elements of $\mathcal{H}$, in a way analogous to dynamical systems. The associated evolution PDE for $u$ is replaced globally by a statement of the form

$$
\frac{d u(t)}{d t} \in-\partial F(u(t)) \text { for all } t>0
$$

with the initial condition $u(0)=u_{0} \in \mathcal{H}$. A known theorem of convex analysis asserts that there exists a unique (sufficiently smooth) $u(t)$ in $\mathcal{H}$ for all $t>0$ provided the functional $F$ satisfies certain conditions such as appropriate convexity [17].

In particular, evolution PDE (4.7) for evaporation-condensation can be recast to form (B.2), where $u=h$ is of bounded variation and $F(h)=\nu \Omega E(h)=\iint \gamma(\nabla h) d A$, the singular surface free energy (1.4). The subgradient $\partial F(h)$ extends the variational derivative of $F(h)$ to the facet $(\nabla h=0)$. A characterization theorem for subgradient systems states that for such a functional $F$, a function $f$ belongs to $\partial F(h)$ if and only if there is a pair of continuous vector-valued functions $\boldsymbol{\xi}_{1}$ and $\boldsymbol{\xi}_{2}$ in $\mathbb{R}^{2}$ satisfying [17]

$$
f=\nu \Omega g_{1} \operatorname{div}\left(\boldsymbol{\xi}_{1}+g \boldsymbol{\xi}_{2}\right),
$$

where $\boldsymbol{\xi}_{1}$ is an element of $\partial J_{1}(\nabla h)$ and $\boldsymbol{\xi}_{2}$ is an element of $\partial J_{2}(\nabla h)$ with $J_{1}(\mathbf{p})=|\mathbf{p}|$ and $J_{2}(\mathbf{p})=|\mathbf{p}|^{3} / 3$. This characterization is central in this framework, with direct implications to boundary conditions at the facet. By virtue of

$$
\partial J_{1}(\mathbf{p})=\left\{\begin{array}{ll}
\{\mathbf{p} /|\mathbf{p}|\} & \text { if } p \neq 0, \\
B^{2}(0,1) & \text { if } p=0,
\end{array} \quad \partial J_{2}(\mathbf{p})=\{|\mathbf{p}| \mathbf{p}\}\right.
$$

one can assert that $\left|\boldsymbol{\xi}_{1}\right| \leq 1$ and $\boldsymbol{\xi}_{2}=0$ for $\mathbf{p}=0$; therefore, $|\xi| \leq 1$ on the facet.

In conclusion, by (B.2)-(B.4), there exists a continuous vector-valued $\boldsymbol{\xi}$ such that

$$
\partial_{t} h=-\nu \Omega g_{1} \operatorname{div} \boldsymbol{\xi} \quad \text { everywhere },
$$

where $g_{1} \boldsymbol{\xi}$ belongs to $\partial \gamma(\mathbf{p})$ for $\mathbf{p}=\nabla h$. In our radial setting, $|\boldsymbol{\xi}| \leq 1$ for $r<r_{\mathrm{f}}(t)$ and $\boldsymbol{\xi}_{2}$ is zero on the facet. These considerations lead to boundary conditions (5.3) and (5.5) for $g>0$. Since $m$ is continuous for $g>0$, so is $h$. (For $g=0$, this argument needs to be modified since $m$ ceases to be continuous [27].) 
Appendix C. Step collapse times for noninteracting steps $(g=0)$. In this appendix, we solve analytically, for both M1 and M2, equations of motion (2.12) with $g=0$ for the two top steps. Our goal is to obtain a recursion relation for the time differences $\delta_{n}:=t_{n}-t_{n-1}$ for each choice of the factor $\mathcal{G}_{i}$. The analytical results enable us to (i) check the accuracy of our numerics for the step ODEs and (ii) explore limitations of empirical formula (5.7). We employ units with $\nu \Omega g_{1}=1=a$.

C.1. Case of M1 $\left(\mathcal{G}_{i}(t) \equiv \mathbf{1}\right)$. First, consider times $t_{n-1}<t<t_{n}$ for fixed collapse number $n(n \geq 1)$. For $g=0$ and $\mathcal{G}_{i} \equiv 1,(2.12)$ reduces to

$$
\dot{r}_{i}=-\frac{r_{i}-r_{i-1}}{r_{i}}, \quad i \geq n .
$$

In particular, for $i=n$ we have $\dot{r}_{n}=-1$ by which

$$
r_{n}(t)=t_{n}-t, \quad t_{n-1}<t \leq t_{n} .
$$

We proceed to determine $r_{n+1}(t)$, which satisfies $r_{n+1} \dot{r}_{n+1}=-r_{n+1}+t_{n}-t$ in view of (C.2). We seek a solution in parametric form by using another independent variable, say, $\tau$. Let $t_{n}-t \equiv \sigma(\tau)$ and $r_{n+1}(t) \equiv \sigma(\tau) p(\tau)$, where $\sigma$ and $p$ are to be determined. The ODE for $r_{n+1}(t)$ yields

$$
\frac{\dot{\sigma}}{\sigma}=-\frac{\dot{p} p}{p^{2}-p+1}
$$

which can be integrated exactly; $\dot{\sigma} \equiv d \sigma / d \tau$. By setting $p(\tau)=\tau$ (without loss of generality), we find $\sigma(\tau)$ and thereby compute $t$ and $r_{n+1}$ as functions of $\tau$ :

$$
\begin{aligned}
r_{n+1}(t(\tau)) & =C\left(\tau^{2}-\tau+1\right)^{-1 / 2} \tau e^{\mathcal{K}(\tau)}, \\
t(\tau) & =t_{n}-C\left(\tau^{2}-\tau+1\right)^{-1 / 2} e^{\mathcal{K}(\tau)},
\end{aligned}
$$

where $\tau>\tau_{*}=\tau_{*, n}$ and

$$
\mathcal{K}(\tau)=\frac{1}{2 \sqrt{3}} \tan ^{-1}\left[\frac{\sqrt{3}(1-2 \tau)}{1+2 \tau-2 \tau^{2}}\right] .
$$

The in principle $n$-dependent $C$ and $\tau_{*}$ are determined by the initial conditions $t\left(\tau_{*}\right)=$ $t_{n-1}$ and $r_{n+1}\left(t\left(\tau_{*}\right)\right)=r_{n+1}\left(t_{n-1}\right) \equiv \mathfrak{R}_{n}$. Thus, we obtain

$$
\begin{aligned}
t(\tau) & =t_{n}-\delta_{n}\left(\frac{\tau_{*}^{2}-\tau_{*}+1}{\tau^{2}-\tau+1}\right)^{1 / 2} e^{\mathcal{K}(\tau)-\mathcal{K}\left(\tau_{*}\right)}, \\
r_{n+1}(t(\tau)) & =\delta_{n}\left(\frac{\tau_{*}^{2}-\tau_{*}+1}{\tau^{2}-\tau+1}\right)^{1 / 2} \tau e^{\mathcal{K}(\tau)-\mathcal{K}\left(\tau_{*}\right)},
\end{aligned}
$$

where

$$
\tau_{*}=\tau_{*, n}=\frac{\Re_{n}}{\delta_{n}} .
$$

As the $n$th step collapses, $t \uparrow t_{n}$ and $\tau \rightarrow \infty$; the radius $r_{n+1}\left(t_{n}\right)$ follows from (C.6).

Now consider times $t_{n}<t<t_{n+1}$ after the $n$th step collapses. Then, $r_{n+1}(t)=$ $t_{n+1}-t$. By continuity of $r_{n+1}(t)$ and use of (C.6), we find the recursion relation

$$
\frac{\delta_{n+1}}{\delta_{n}}=\sqrt{\tau_{*, n}^{2}-\tau_{*, n}+1} e^{-\mathcal{K}\left(\tau_{*, n}\right)},
$$

where $\tau_{*, n} \equiv \tau_{*}=\mathfrak{R}_{n} / \delta_{n}=r_{n+1}\left(t_{n-1}\right) / \delta_{n}$. 
It is of interest to discuss implications of (C.8) in the limit $n \rightarrow \infty$ under the assumption that $\delta_{n+1} / \delta_{n} \gtrsim 1$. By (C.8), $\tau_{*, n}$ cannot approach 0. If, in addition, $\delta_{n+1} / \delta_{n}$ is assumed to be bounded with $n$, as is presumably the case for an initial conical profile (where $r_{i}(0)$ in linear in $i$ ), we assert that $\tau_{*, n}$ must approach a finite value: $\tau_{*, n} \rightarrow \tau_{\diamond}$ as $n \rightarrow \infty$. Thus, this $\tau_{\diamond}$ obeys

$$
\left(\tau_{\diamond}^{2}-\tau_{\diamond}+1\right)^{1 / 2} e^{-\mathcal{K}\left(\tau_{\diamond}\right)}=1
$$

By numerically solving this transcendental equation, we find $\tau_{\diamond} \approx 1.66$, in agreement with our (independent) numerical simulations for (2.12) with $\mathcal{G}_{i}=1$.

C.2. Case of M2. Next, we study (2.12) with $g=0$ and $\mathcal{G}_{i} \equiv\left(r_{i}+r_{i-1}\right) / 2 r_{i}$. First, consider $t_{n-1}<t<t_{n}$ and fixed $n(n \geq 1)$. The step ODEs become

$$
\dot{r}_{i}=-\frac{r_{i}+r_{i-1}}{2 r_{i}} \frac{r_{i}-r_{i-1}}{r_{i}}, \quad i \geq n .
$$

In particular, for $i=n$ we have $\dot{r}_{n}=-1 / 2$, by which

$$
r_{n}(t)=\frac{1}{2}\left(t_{n}-t\right), \quad t_{n-1}<t \leq t_{n} .
$$

We proceed to determine $r_{n+1}(t)$, which satisfies

$$
8 r_{n+1}^{2} \dot{r}_{n+1}=-\left(2 r_{n+1}+t_{n}-t\right)\left[2 r_{n+1}-\left(t_{n}-t\right)\right]
$$

in view of (C.11). Again, we seek a solution in parametric form by using an independent variable, $\tau$. By employing definitions of Appendix C.1, we find

$$
\begin{aligned}
t(\tau) & =t_{n}-\delta_{n} e^{\mathcal{K}(\tau)-\mathcal{K}\left(\tau_{*}\right)}, \\
r_{n+1}(t(\tau)) & =\delta_{n} \tau e^{\mathcal{K}(\tau)-\mathcal{K}\left(\tau_{*}\right)},
\end{aligned}
$$

where $\tau>\tau_{*}=\tau_{*, n}$ (as in Appendix C.1) and

$$
\mathcal{K}(\tau)=-\sum_{\mathfrak{s}_{i} \in \mathcal{I}} \frac{\mathfrak{s}_{i}}{3 \mathfrak{s}_{i}-1} \ln \left(\tau-\mathfrak{s}_{i}\right), \quad \mathcal{I}=\left\{\mathfrak{s}: 8 \mathfrak{s}^{3}-4 \mathfrak{s}^{2}+1=0\right\}
$$

cf. (C.5) and recall $\tau_{*}=\tau_{*, n}=\mathfrak{R}_{n} / \delta_{n}$. As the $n$th step collapses, $\tau \rightarrow \infty$; the radius $r_{n+1}\left(t_{n}\right)$ is obtained from (C.12).

Now consider times after the $n$th collapse, $t_{n}<t<t_{n+1}$; then, $r_{n+1}(t)=$ $\frac{1}{2}\left(t_{n+1}-t\right)$. By continuity of $r_{n+1}(t)$ and (C.12), we find the recursion relation

$$
\frac{\delta_{n+1}}{\delta_{n}}=2 \prod_{\mathfrak{s}_{i} \in \mathcal{I}}\left(\tau_{*, n}-\mathfrak{s}_{i}\right)^{\frac{\mathfrak{s}_{i}}{3 \mathfrak{s}_{i}-1}} ; \quad \tau_{*, n}=r_{n+1}\left(t_{n-1}\right) / \delta_{n} .
$$

By (C.14), $\tau_{*, n}$ cannot approach 0 . If $\delta_{n+1} / \delta_{n}$ is again assumed to be bounded with $n$, we can take $\tau_{*, n} \rightarrow \tau_{\diamond}$ as $n \rightarrow \infty$, where

$$
2 \prod_{\mathfrak{s}_{i} \in \mathcal{I}}\left(\tau_{\diamond}-\mathfrak{s}_{i}\right)^{\frac{\mathfrak{s}_{i}}{3_{i}-1}}=1 ;
$$

cf. (C.9) for $\mathcal{G}_{i} \equiv 1$. By numerically solving this transcendental equation, we find $\tau_{\diamond} \simeq 0.940$, in agreement with our step simulations for (2.12). Using this result with $t \approx t_{n} \rightarrow \infty$, we find that the jump of the flux $\boldsymbol{\xi}$ entering (5.6) stems from

$$
\widetilde{\mathcal{G}}(t) \sim \lim _{t_{n} \rightarrow \infty} \frac{r_{n+2}\left(t_{n}\right)+r_{n+1}\left(t_{n}\right)}{2 r_{n+2}\left(t_{n}\right)} \simeq 0.766
$$

Copyright $@$ by SIAM. Unauthorized reproduction of this article is prohibited. 
Appendix D. Continuum solutions for $\boldsymbol{g}=\mathbf{0}$. In this appendix, we provide some detailed derivations of formulas needed in section 5.4.

Consider the PDE solution (5.11). The differentiation of height continuity, (5.2), with respect to $t$ under initial data (5.8) entails

$$
\dot{h}_{\mathrm{f}}(t)=-\dot{r}_{\mathrm{f}}(t)-\frac{1}{r_{\mathrm{f}}(t)}+\frac{t}{r_{\mathrm{f}}(t)^{2}} \dot{r}_{\mathrm{f}}(t) .
$$

Accordingly, generalized condition $(5.6)$ on $\xi(\cdot, t)$ yields an ODE for $r_{\mathrm{f}}(t)$ :

$$
\dot{r}_{\mathrm{f}}(t)=\frac{[2 \widetilde{\mathcal{G}}(t)-1] r_{\mathrm{f}}}{r_{\mathrm{f}}^{2}-t} ; \quad r_{\mathrm{f}}(0)=r_{\mathrm{f} 0}
$$

Once $r_{\mathrm{f}}(t)$ is computed, $h_{\mathrm{f}}(t)$ can be found via (5.2) and (5.11).

Next, specify values of $\widetilde{\mathcal{G}}(t)$ by Remark 5.1 . For $\widetilde{\mathcal{G}}=1, r_{\mathrm{f}}(t)$ satisfies

$$
\dot{r}_{\mathrm{f}}=\frac{r_{\mathrm{f}}}{r_{\mathrm{f}}^{2}-t} ; \quad r_{\mathrm{f}}(0)=r_{\mathrm{f} 0} .
$$

This ODE is solved exactly by inversion, $r_{\mathrm{f}} \mapsto T\left(r_{\mathrm{f}}\right)=t$ :

$$
t=T\left(r_{\mathrm{f}}\right)=-\left(r_{\mathrm{f0}}^{3} / 3\right) r_{\mathrm{f}}^{-1}+r_{\mathrm{f}}^{2} / 3 .
$$

Hence, $r_{\mathrm{f}}$ satisfies the cubic polynomial equation $r_{\mathrm{f}}^{3}-3 t r_{\mathrm{f}}-r_{\mathrm{f} 0}^{3}=0$ and turns out to be $[3] r_{\mathrm{f}}(t)=\left[r_{\mathrm{f} 0}^{3} / 2+\vartheta(t)\right]^{1 / 3}+\left[r_{\mathrm{f} 0}^{3} / 2-\vartheta(t)\right]^{1 / 3}$, where $\vartheta(t)=\sqrt{r_{\mathrm{f0}}^{6} / 4-t^{3}}$. (The positive square root is taken for $t<2^{-2 / 3} r_{\mathrm{f} 0}^{2}$.) For $t>2^{-2 / 3} r_{\mathrm{f} 0}^{2}$, the solution reads

$$
r_{\mathrm{f}}(t)=2 \sqrt{t} \cos \left(\frac{1}{3} \tan ^{-1} \frac{\sqrt{4 t^{3}-r_{\mathrm{f} 0}^{6}}}{r_{\mathrm{f} 0}^{3}}\right) .
$$

Equation (D.5) leads to asymptotic formula (5.13). The facet height is furnished by height continuity, $h_{\mathrm{f}}(t)=h_{\mathrm{f} 0}+r_{\mathrm{f} 0}-r_{\mathrm{f}}-t / r_{\mathrm{f}}$; in particular, we wind up with (5.14).

Now set $\widetilde{\mathcal{G}}(t)=\mathfrak{c} \neq 1$. Then, again by inversion, (D.2) is exactly solved by

$$
t=T\left(r_{\mathrm{f}}\right)=(4 \mathfrak{c}-1)^{-1} r_{\mathrm{f}}^{2}\left[1-\left(\frac{r_{\mathrm{f} 0}}{r_{\mathrm{f}}}\right)^{(4 \mathfrak{c}-1) /(2 \mathfrak{c}-1)}\right] .
$$

This equation no longer leads to a polynomial in $r_{\mathrm{f}}$, in contrast to (D.4). We have been unable to invert (D.6) analytically. Nonetheless, we can obtain an asymptotic formula for $r_{\mathrm{f}}$ in the limit $t \rightarrow \infty$. Notably, growth of a real, nonnegative $r_{\mathrm{f}}(t)$ as $t \rightarrow \infty$ implies $\mathfrak{c}>1 / 2$. For these values of $\mathfrak{c}$, the first term on the right-hand side of (D.6) dominates for large enough $t$. A more precise argument to deduce the growth of $r_{\mathrm{f}}(t)$ can be sketched as follows. As $t \rightarrow \infty$, we have either $r_{\mathrm{f}} \rightarrow+\infty$ or $r_{\mathrm{f}} \downarrow 0$ (since $\left.r_{\mathrm{f}} \geq 0\right)$. Suppose that $r_{\mathrm{f}}(t)$ decays as $t \rightarrow \infty$. Consider large $t$. For $\mathfrak{c}<1 / 4$, (D.6) would entail $(1-4 \mathfrak{c}) t \sim r_{\mathrm{f} 0}^{(4 \mathfrak{c}-1) /(2 \mathfrak{c}-1)} r_{\mathrm{f}}^{1 /(1-2 \mathfrak{c})}$, a contradiction. A similar contradiction is encountered if $1 / 4 \leq \mathfrak{c}<1 / 2$. For $\mathfrak{c}>1 / 2$, by (D.6) $r_{\mathrm{f}}(t)$ would not be compatible with a real solution. Therefore, $r_{\mathrm{f}}(t) \rightarrow+\infty$ as $t \rightarrow \infty$, which implies (5.16) with $\mathfrak{c}>1 / 2$. In the exceptional case with $\mathfrak{c}=1 / 2$, by (D.2) $r_{\mathrm{f}}(t) \equiv r_{\mathrm{f} 0}$ for all $t \geq 0$; there is no facet evolution. 
Appendix E. Formal boundary layer analysis. In this appendix, we provide heuristic derivations of formulas presented in section 6.3 by invoking elements of singular perturbation theory [22]. Self-similarity is not needed for our main arguments.

First, $m(r, t)$ is recast to a form that manifests the boundary layer width. Inside the boundary layer (inner region), write

$$
m(r, t)=a_{0}(t) f_{0}(\zeta, t), \quad \zeta=\frac{r-r_{\mathrm{f}}(t)}{g^{\alpha} w(t)}=\mathcal{O}(1) .
$$

The substitution of (E.1) into PDE (4.9) yields

$$
\begin{aligned}
\dot{a}_{0} f_{0} & +a_{0} \partial_{t} f_{0}-g^{-\alpha} \frac{a_{0}}{w}\left(\dot{r}_{\mathrm{f}}+g^{\alpha} \dot{w} \zeta\right) \partial_{\zeta} f_{0} \\
= & -\frac{1}{\left(r_{\mathrm{f}}+g^{\alpha} w \zeta\right)^{2}} \\
& +g^{1-2 \alpha} \frac{a_{0}^{2}}{w^{2}} \partial_{\zeta}\left\{\left(r_{\mathrm{f}}+g^{\alpha} w \zeta\right)^{-1} \partial_{\zeta}\left[\left(r_{\mathrm{f}}+g^{\alpha} w \zeta\right) f_{0}^{2}\right]\right\} .
\end{aligned}
$$

By treating $\zeta$ as well as $a_{0}, f_{0}, w, r_{\mathrm{f}}$, and their derivatives as $\mathcal{O}(1)$ quantities for $0<g \ll 1$, we observe that the $\mathcal{O}\left(g^{-\alpha}\right)$ term on the left-hand side of the last equation must be balanced by the $\mathcal{O}\left(g^{1-2 \alpha}\right)$ term; thus, $\alpha=1$, as claimed in (6.6).

The resulting equation for $f_{0}$ reads

$$
\partial_{\zeta \zeta}\left(f_{0}^{2}\right)=-\ell \partial_{\zeta} f_{0} \quad \zeta>0 ; \quad \ell:=w \dot{r}_{\mathrm{f}} / a_{0} .
$$

Now let us match $a_{0}(t) f_{0}(\zeta, t)$ as $\zeta \rightarrow \infty$ with the outer solution for $m$ given by (5.12) as $r \downarrow r_{\mathrm{f}}(t)$, assuming there is a region where the two solutions overlap. Accordingly, we choose to set $a_{0}(t)$ equal to $1-t / r_{\mathrm{f}}^{2}$, as displayed in (6.7), while we take

$$
f_{0} \rightarrow 1 \text { as } \zeta \rightarrow \infty \text {. }
$$

By integrating (E.2), in view of (E.3), we obtain

$$
\left(f_{0}^{2}\right)_{\zeta}=\ell\left(1-f_{0}\right), \quad \zeta>0 ; \quad f_{0}=0 \quad \text { as } \zeta \downarrow 0 .
$$

Here, we impose the condition of slope continuity at the facet edge. We henceforth consider $\partial_{t} f_{0} \equiv 0$ and set $\ell=$ const. By (E.4), we obtain $2 f_{0}+\ln \left(1-f_{0}\right)=-\ell \zeta$, which must be reconciled with (E.3). Thus, $\ell>0$; without loss of generality, set $\ell=1$. This value leads to formula (6.8). It is of some interest to note that $f_{0}(\zeta)=$ $1+W\left(-e^{-1-\zeta / 2}\right)$, where $W(x)$ is the Lambert function [8].

By definition of $\ell$ in (E.2), we extract a formula for the boundary layer width in accord with (6.6). This finding concludes our computation of the inner solution for $m$. As usual, a composite formula can be constructed by adding the outer solution (5.12) to the inner solution, $a_{0}(t) f_{0}(\zeta)$, and subtracting their common limit (valid in the overlap region where $\zeta \rightarrow \infty$ ) [22]; cf. (6.7).

Appendix F. Proof of convergence of step schemes for $\boldsymbol{g}=\mathbf{0}$. In this appendix, we provide a proof of Proposition 7.1 by invoking the formalism of the LaxWendroff theorem [30]. For ease of notation, we suppress dependencies that are not explicitly used, i.e., let $U^{a}(t)=\left(U_{j}(t)\right)_{j \in\{i \in \mathbb{Z} \mid i \leq N\}}$ and $F_{j}(t)=F\left(U^{a}(t) ; j\right)$ (where $\mathbb{Z}$ : the set of integers). Below, $\left\|u-U^{a}\right\|_{\mathcal{X}}$ means the error, by the metric of space $\mathcal{X}$, in the approximation of $u(\chi, t)$ by the piecewise constant function with values $U_{j}(t)$ for $\chi \in\left(\chi_{j-1 / 2}, \chi_{j+1 / 2}\right)$; also, $\varphi_{z}$ means $\partial_{z} \varphi$ for $z=\chi, t$. We use the notions of total variation (TV) and TV-stability, defined in [31]. 
Before we proceed to the main proof of Proposition 7.1, it is advisable to address the sense by which solutions of our discrete schemes may converge.

Lemma F.1. For a compactly supported initial condition (i.e., a finite height profile) that is monotone increasing within its support, the discrete dynamics for $M 1$ and $M 2$ are TV diminishing with time. In particular, the $U^{a}$ is $T V$-stable.

Proof. From section 3, if the initial data is monotone increasing in $j$, the dynamical system preserves the step ordering; in fact, steps do not collide. Therefore, for a class of dynamics with appropriate initial conditions including linear $r_{j}(0), \dot{U}_{j}(t) \leq 0$ and $U_{j}(t)$ is always nonincreasing with respect to time for all $j$. This fact together with the monotonicity of $\left\{U_{j}\right\}$ implies $T V(U(t)) \leq T V(U(0))<\infty$ (by telescoping).

As a consequence, the family $\left\{U^{a}(t)\right\}$ is $L^{1}$-compact for any $a \rightarrow 0, t \in[0, T]$ and fixed $T$ [31]. Thus, there exists an $L^{1}$-convergent sequence in $\left\{U^{a}\right\}$, which we denote by $\left(U^{a}\right)$ with a slight abuse of notation, for each of M1 and M2 $(\mathfrak{b}=1 / 2,2 / 3)$. Without proof, we claim that this property is true for $0<\mathfrak{b} \leq 1$ in $(7.5)$ and use it in the proof of Proposition 7.1. We improve the above convergence result by showing that all convergent sequences approach the same entropy solution of a specific (bdependent) conservation law.

Proof of Proposition 7.1. First, we show that every convergent sequence in $\left\{U^{a}(t)\right\}$ $L^{1}$-converges to a weak solution of conservation law (7.2). Our proof is similar to the ones for the Lax-Wendroff theorem [30,31], except that for M1 and M2, we proved above (not simply assumed) existence of convergent sequences in $\left\{U^{a}\right\}$. Fix $T \in[0, \infty)$ and consider a sequence in $\left\{U^{a}\right\}$ that converges to some $u \in L^{1}\left(\left(-\infty, h_{f}(0)\right] \times[0, T]\right)$. With a slight abuse of notation, we denote elements of this sequence by $U^{a}$ with components $U_{j}^{a}=U_{j}$. Let $\varphi \in C_{0}^{\infty}\left(\left(-\infty, h_{f}(0)\right] \times[0, T)\right)$ be a test function. We assert that

$$
\begin{aligned}
& a \int_{0}^{T} \sum_{j} \varphi_{t}\left(\chi_{j}, t\right) U_{j} \mathrm{~d} t-a \sum_{j} \varphi\left(\chi_{j}, 0\right) U_{j}(0) \\
& \quad=-a \int_{0}^{T} \sum_{j} \varphi\left(\chi_{j}, t\right) \dot{U}_{j}(t) \mathrm{d} t \\
& \quad=a \int_{0}^{T} \sum_{j} \frac{1}{a} \varphi\left(\chi_{j}, t\right)\left[F_{j}(t)-F_{j-1}(t)\right] \mathrm{d} t \\
& \quad=-a \int_{0}^{T} \sum_{j} \frac{\varphi\left(\chi_{j+1}, t\right)-\varphi\left(\chi_{j}, t\right)}{a} F_{j}(t) \mathrm{d} t
\end{aligned}
$$

via summation by parts; boundary terms vanish since $\varphi$ is compactly supported. The leftmost side (top line) of (F.1) converges to $\int_{0}^{T} \int_{-\infty}^{\infty} \varphi_{t}(\chi, t) u(\chi, t) \mathrm{d} \chi \mathrm{d} t-$ $\int_{-\infty}^{\infty} \varphi(\chi, 0) u(\chi, 0) \mathrm{d} \chi$ in view of boundedness of $\varphi$ (and $\left.\varphi_{t}\right)$. Below, we show that the right-hand side (bottom line) of (F.1) converges to $-\int_{0}^{T} \int_{-\infty}^{\infty} \varphi_{\chi}(\chi, t) f(u(\chi, t)) \mathrm{d} \chi \mathrm{d} t$ :

$$
\begin{aligned}
\int_{0}^{T} & {\left[\int_{-\infty}^{\infty} \varphi_{\chi}(\chi, t) f(u(\chi, t)) d \chi-a \sum_{j} \frac{\varphi\left(\chi_{j+1}\right)-\varphi\left(\chi_{j}\right)}{a} F_{j}(t)\right] \mathrm{d} t } \\
& =\sum_{j} \int_{0}^{T} \int_{\chi_{j}}^{\chi_{j+1}}\left[\varphi_{\chi}(\chi, t) f(u(\chi, t))-\frac{\varphi\left(\chi_{j+1}\right)-\varphi\left(\chi_{j}\right)}{a} F_{j}(t)\right] \mathrm{d} \chi \mathrm{d} t ;
\end{aligned}
$$

Copyright $@$ by SIAM. Unauthorized reproduction of this article is prohibited. 
the terms on the right-hand side are rearranged to yield

$$
\begin{aligned}
& \sum_{j} \int_{0}^{T} \int_{\chi_{j}}^{\chi_{j+1}} \varphi_{\chi}\left[f(u(\chi, t))-F_{j}(t)\right] \mathrm{d} \chi \mathrm{d} t \\
& \quad+\sum_{j} \int_{0}^{T} \int_{\chi_{j}}^{\chi_{j+1}}\left[\varphi_{\chi}(\chi, t)-\frac{\varphi\left(\chi_{j+1}\right)-\varphi\left(\chi_{j}\right)}{a}\right] F_{j}(t) d \chi \mathrm{d} t,
\end{aligned}
$$

recalling that $f$ coincides with $F$. Since $F_{j}$ is bounded in $\operatorname{supp}(\varphi)$, the second term in (F.3) converges to 0 . The first term is bounded by

$$
\begin{aligned}
& \left\|\partial_{\chi} \phi\right\|_{L^{\infty}} \sum_{j} \int_{0}^{T} \int_{\chi_{j}}^{\chi_{j+1}}\left|f(u(\chi, t))-F_{j}(t)\right| \mathrm{d} \chi \mathrm{d} t \\
& \leq C \int_{0}^{T}\left\|u-U^{a}\right\|_{L_{\chi}^{\mathfrak{b}}} \mathrm{d} t \leq \widetilde{C}\left\|u-U^{a}\right\|_{L^{1}(\mathbb{R} \times[0, T])},
\end{aligned}
$$

where $\widetilde{C}$ is a constant depending on the Hölder exponent, $\mathfrak{b}$, of $F$; also, the embedding $L^{\mathfrak{b}} \subset L^{1}$ on a compact set was used. Because $\left\|u-U^{a}\right\|_{L^{1}} \rightarrow 0$, we conclude that $u$ is a weak solution of $(7.2)$ with $\mathfrak{b}=\varsigma /(\varsigma+1)$.

Next, we show that $\left\{U^{a}\right\}$ satisfies a (semidiscrete) entropy condition [31]. Let $\eta(\cdot) \in C^{\infty}\left(\left(-\infty, h_{f}(0)\right]\right)$ be any convex function ${ }^{7}$ and $\psi(\cdot)$ be its entropy flux function, i.e., $\psi^{\prime}(u)=\eta^{\prime}(u) f^{\prime}(u)\left[31\right.$, where the prime denotes differentiation in $u$ and $f^{\prime} \in$ $L^{1}\left(\left(-\infty, h_{f}(0)\right]\right)$ is interpreted as a weak derivative. Accordingly, we have

$$
\begin{aligned}
-\frac{1}{a}\left[\psi\left(U_{j}\right)-\psi\left(U_{j-1}\right)\right] & =-\frac{1}{a} \int_{U_{j-1}}^{U_{j}} \psi^{\prime}(u) \mathrm{d} u=-\frac{1}{a} \int_{U_{j-1}}^{U_{j}} \eta^{\prime}(u) f^{\prime}(u) \mathrm{d} u \\
& \geq-\frac{\eta^{\prime}\left(U_{j}\right)}{a} \int_{U_{j-1}}^{U_{j}} f^{\prime}(u) \mathrm{d} u=\eta^{\prime}\left(U_{j}\right) \dot{U}_{j}(t)=\partial_{t} \eta\left(U_{j}\right) .
\end{aligned}
$$

The application of a procedure similar to the one applied to show the weak convergence of the solution leads to the entropy inequality $\partial_{t} \eta(u)+\partial_{\chi} \psi(u) \leq 0$ in the weak sense [31]. Because all convergent sequences in the family $\left\{U^{a}\right\}$ approach the same limit, we conclude that $\left\{U^{a}\right\}$ weakly converges to the entropy solution.

It is worth noting that the finite support of the initial data does not affect our proof, because each discrete scheme is backward in $j$ for $g=0$. This property ceases to hold for $g>0$.

Acknowledgments. This article is dedicated to the memory of the late Professor John E. Osborn for his valuable advice on numerical methods at initial stages of this work. We are indebted to Professors Yoshikazu Giga, Robert V. Kohn, Ricardo Nochetto, and Olivier Pierre-Louis for stimulating discussions and to two anonymous reviewers for their constructive remarks. Special thanks are due to Mr. Joshua Schneider for help with the numerics, particularly for Figure 6.7. The first author (K. Nakamura) is grateful to the University of Maryland for a Graduate Student Summer Research Fellowship during the summer of 2011, when part of this work was completed.

\footnotetext{
${ }^{7}$ This function $\eta(u)$ should not be confused with the similarity variable $\eta=r / \sqrt{t}$ of section 6 .
} 


\section{REFERENCES}

[1] H. Al Haju Shehadeh, The Evolution of a Crystal Surface: Step ODEs, PDEs and Selfsimilarity, Ph.D. thesis, Courant Institute, New York University, New York, 2010.

[2] H. Al Haju Shehadeh, R. V. Kohn, and J. Weare, The evolution of a crystal surface: Analysis of a one-dimensional step train connecting two facets in the ADL regime, Physica D, 240 (2011), pp. 1771-1784.

[3] G. Birkhoff and S. Maclane, A Survey of Modern Algebra, MacMillan, New York, 1959.

[4] H. P. Bonzel, E. Preuss, And B. Steffen, The dynamical behavior of periodic surface profiles on metals under the influence of anisotropic surface-energy, Appl. Phys. A Mater., 35 (1984), pp. 1-8.

[5] W. K. Burton, N. Cabrera, and F. C. Frank, The growth of crystals and the equilibrium structure of their surfaces, Philos. Trans. R. Soc. London Ser. A, 243 (1951), pp. 299-358.

[6] A. Chame, S. Rousset, H. P. Bonzel, and J. Villain, Slow dynamics of stepped surfaces, Bulgarian Chem. Commun., 29 (1996/97), pp. 398-434.

[7] E. A. Coddington and N. Levinson, Theory of Ordinary Differential Equations, McGrawHill, Boston, 1955, pp. 13-15.

[8] R. M. Corless, G. H. Gonnet, D. E. G. Hare, D. J. Jeffrey, and D. E. Knuth, On the Lambert $W$ function, Adv. Comput. Math., 5 (1996), pp. 329-359.

[9] W. E And N. K. YIP, Continuum theory of epitaxial growth. I, J. Statist. Phys., 104 (2001), pp. 221-253.

[10] G. Ehrlich and F. Hudda, Atomic view of surface diffusion: Tungsten on Tungsten, J. Chem. Phys., 44 (1966), pp. 1039-1099.

[11] P.-W. FoK, Simulation of Axisymmetric Stepped Surfaces with a Facet, Ph.D. thesis, Massachusetts Institute of Technology, Cambridge, MA, 2006.

[12] P.-W. Fok, R. R. Rosales, and D. Margetis, Unification of step bunching phenomena on vicinal surfaces, Phys. Rev. B, 76 (2007), 033408.

[13] P.-W. Fok, R. R. Rosales, And D. Margetis, Facet evolution on supported nanostructures: Effect of finite height, Phys. Rev. B, 78 (2008), 235401.

[14] Y. GIGA, Shocks and very strong vertical diffusion, in Lectures on Partial Differential Equations: Proceedings in Honor of Louis Nirenberg's 75th Birthday, New Studies in Advance Mathematics, Vol. 2, S.-Y. A. Chang, C.-S. Lim, and H.-T. Yau, eds., International Press, Somerville, MA, 2003, pp. 95-102.

[15] Y. Giga, Singular diffusivity-facets, shocks and more, in Applied Mathematics Entering the 21st Century, Invited Talks from the ICIAM 2003 Congress, J. M. Hill and R. Moore, eds., SIAM, Philadelphia, 2003, pp. 121-138.

[16] M.-H. GigA AND Y. GIGA, Minimal vertical singular diffusion preventing overturning for the Burgers equation, in Recent Advances in Scientific Computing and Partial Differential Equations, Contemp. Math. 330, AMS, Providence, RI, 2003, pp. 73-88.

[17] M.-H. Giga, Y. Giga, and R. Kobayashi, Very singular diffusion equations, Adv. Stud. Pure Math., 31 (2001), pp. 93-126.

[18] E. E. Gruber and W. W. Mullins, On the theory of anisotropy of crystalline surface tension, J. Phys. Chem. Solids, 28 (1967), pp. 875-887.

[19] J. K. HaLe, Ordinary Differential Equations, Willy-Interscience, Providence, RI, 1969, pp. 1222.

[20] C. Herring, Effect of change of scale on sintering phenomena, J. Appl. Phys., 21 (1950), pp. 301-303.

[21] C. Herring, Some theories on the free energies of crystal surfaces, Phys. Rev., 82 (1951), pp. 87-93.

[22] M. H. Holmes, Introduction to Perturbation Methods, Springer, New York, 1998.

[23] B. R. Hunt, R. L. Lipsman, J. E. Osborn, And J. Rosenberg, Differential Equations with Matlab, Wiley, New York, 2005.

[24] N. Israeli and D. Kandel, Profile of a decaying crystalline cone, Phys. Rev. B, 60 (1999), pp. 5946-5962.

[25] N. Israeli and D. Kandel, Decay of one-dimensional surface modulations, Phys. Rev. B, 62 (2000), pp. 13707-13717.

[26] H.-C. Jeong and E. D. Williams, Steps on surfaces: Experiments and theory, Surf. Sci. Rep., 34 (1999), pp. 171-294.

[27] R. Kobayashi and Y. Giga, Equations with singular diffusivity, J. Statist. Phys., 95 (1999), pp. $1187-1220$.

[28] R. V. Kohn, T. S. Lo, And N. K. Yip, Continuum limit of a step flow model of epitaxial growth, in Statistical Mechanical Modeling in Materials Science, MRS Symposia Proceedings 701, 
M. C. Bartelt, J. W. Evans, A. S. Karma, S. Torquato, and D. E. Wolf, eds., Materials Research Society, Warrendale, PA, 2002, pp. T1.7.1-T1.7.7.

[29] P. Lax, Hyperbolic Systems of Conservation Laws and the Mathematical Theory of Shock Waves, SIAM, Philadelphia, 1973.

[30] P. Lax and B. Wendroff, Systems of conservation laws, Commun. Pure Appl. Math., 13 (1960), pp. 217-237.

[31] R. J. LeVeque, Numerical Methods for Conservation Laws, 2nd ed., Birkhäuser, Basel, 2005.

[32] V. I. Marchenko and A. Ya. Parshin, Elastic properties of crystal surfaces, Sov. Phys. JETP, 52 (1980), pp. 129-131.

[33] D. Margetis, M. J. Aziz, And H. A. Stone, Continuum approach to self-similarity and scaling in morphological relaxation of a crystal with a facet, Phys. Rev. B, 71 (2005), 165432.

[34] D. Margetis, P.-W. Fok, M. J. Aziz, And H. A. Stone, Continuum theory of nanostructure decay via a microscale condition, Phys. Rev. Lett., 97 (2006), 096102.

[35] D. Margetis and R. V. Kohn, Continuum relaxation of interacting steps on crystal surfaces in $2+1$ dimensions, Multiscale Model. Simul., 5 (2006), pp. 729-758.

[36] D. Margetis And K. NAkAmura, From crystal steps to continuum laws: Behavior near large facets in one dimension, Physica D, 240 (2011), pp. 1100-1110.

[37] T. Michely and J. Krug, Islands, Mounds and Atoms: Patterns and Processes in Crystal Growth Far From Equilibrium, Springer, Berlin, 2004.

[38] C. Misbah, O. Pierre-Louis, and Y. Saito, Crystal surfaces in and out of equilibrium: A modern view, Rev. Mod. Phys., 82 (2010), pp. 981-1040.

[39] W. W. Mullins, The effect of thermal grooving on grain boundary motion, Acta Metall., 6 (1958), pp. 414-427.

[40] R. Najafabadi and J. R. Srolovitz, Elastic step interactions on vicinal surfaces of FCC metals, Surf. Sci., 317 (1994), pp. 221-234.

[41] P. Nozières, On the motion of steps on a vicinal surface, J. Phys. (France), 48 (1987), pp. 1605-1608.

[42] I. V. Odisharia, Simulation and Analysis of the Relaxation of a Crystalline Surface, Ph.D. thesis, Courant Institute, New York University, New York, 2006.

[43] M. Ozdemir And A. ZAngwill, Morphological equilibration of a corrugated crystalline surface, Phys. Rev. B, 42 (1990), pp. 5013-5024.

[44] A. Pimpinelli, V. Tonchev, A. Videcoq, and M. Vladimirowa, Scaling and universality of self-organized patterns on unstable vicinal surfaces, Phys. Rev. Lett., 88 (2002), 206103.

[45] A. Pimpinelli and J. Villain, Physics of Crystal Growth, Cambridge University Press, Cambridge, UK, 1998.

[46] A. Rettori and J. Villain, Flattening of grooves on a crystal surface: A method of investigation of surface roughness, J. Phys. (France), 49 (1988), pp. 257-267.

[47] M. SATo, Effect of step permeability on step instabilities due to alternation of kinetic coeffcients on a growing vicinal face, Eur. Phys. J. B, 59 (2007), pp. 311-318.

[48] J. SCHNEIDER, private communication.

[49] R. L. Schwoebel And E. J. Shipsey, Step motion on crystal surfaces, J. Appl. Phys., 37 (1966), pp. 3682-3686.

[50] W. Selke And P.M. Duxbury, Equilibration of crystal surfaces, Phys. Rev. B, 52 (1995), pp. 17468-17479.

[51] H. Sponn, Surface dynamics below the roughening transition, J. Phys. I (France), 3 (1993), pp. 69-81.

[52] Y.-H. Tsai, Y. GIGA, AND S. Osher, A level set approach for computing discontinuous solutions of Hamilton-Jacobi equations, Math. Comp., 72 (2003), pp. 159-181.

[53] G. N. Watson, A Treatise on the Theory of Bessel Functions, 2nd ed., Cambridge University Press, Cambridge, UK, 1995, pp. 77-78.

Copyright $@$ by SIAM. Unauthorized reproduction of this article is prohibited. 